\title{
SUPOSICIONES DE GRANDES Y DENSAS POBLACIONES PREHISTÓRICAS EN LA AMAZONÍA, EVALUADAS A PARTIR DE LAS EVIDENCIAS ARQUEOLÓGICAS, ETNOGRÁFICAS Y ECOLÓGICAS
}

\author{
Betty J. Meggers \\ Smithsonian Institution, Washington DC
}

Un creciente número de antropólogos ha apoyado la idea de la existencia de densas poblaciones sedentarias a través de la Amazonía, basada principalmente en la presencia de manchas de terra preta a lo largo de ríos principales que testifican la existencia de grandes asentamientos permanentes. Esta interpretación es puesta a prueba por los resultados del reconocimiento arqueológico a lo largo de los ríos Tocantins, Xingú, Jamarí y Essequibo. El uso de criterios uniformes para clasificar cerámica procedente de múltiples excavaciones estratigráficas en sitios habitacionales y la creación de secuencias seriadas, proveen detalladas cronologías relativas en cada región que son complementadas por numerosos fechados radiocarbónicos. Estos datos indican, 1) que la mayoría de sitios son el producto de múltiples reocupaciones por comunidades pequeñas, las cuales con el tiempo incrementaron su tamaño; 2) que pocos sitios fueron ocupados simultáneamente durante cada fase, y 3) que los sitios más tempranos fueron frecuentemente evitados por sitios de fases posteriores, incrementando así el número de manchas de terra preta a través del tiempo, más no el tamaño de la población. Los datos etnográficos comprueban la existencia de este comportamiento entre sobrevivientes de grupos tradicionales, los datos paleoambientales proveen ideas sobre su origen y significación adaptativa.

D uring the past decade, an increasing number of anthropologists have argued for the existence of dense sedentary populations throughout Amazonia based primarily on the presence of patches of terra preta along the principal rivers assumed to equate with large permanent settlements. This interpretation is challenged by the results of archeological survey along the Tocantins, Xingu, Jamari, and Essequibo rivers. The use of uniform criteria for classifying pottery from multiple stratigraphic excavations in habitation sites and for creating seriated sequences provides detailed relative chronologies in each region that are supplemented by numerous radiocarbon dates. These data indicate (1) that most sites are the product of multiple reoccupations by small communities, which increased their dimensions over time; (2) that few sites were occupied simultaneously during each phase, and (3) that earlier sites were frequently avoided by later phases, increasing the number of patches of terra preta through time but not the size of the population. Ethnographic data attest to the existence of this behavior among surviving traditional groups and paleoenvironmental data provide clues to its origin and adaptive significance.

Video Conferencia dictada el 15 de Mayo del 2006 
Desde la llegada de los primeros europeos, la Amazonía ha permanecido como objeto de fascinación. Empezando con las descripciones de Carvajal y Ralegh en el siglo XVI, la ilusión que la floresta exuberante esconde riquezas sin igual ha persistido, no obstante el fracaso de todos los esfuerzos por encontrarlas. Aunque la exploración, colonización, construcción y otras actividades fallaron en encontrar evidencia, los antropólogos siguen apoyando la existencia de grandes establecimientos permanentes a lo largo de los tributarios, de ciudades con miles de habitantes en el Amazonas medio y la isla de Marajó, y un nivel estatal de complejidad social en las Guianas (Fig. 1).

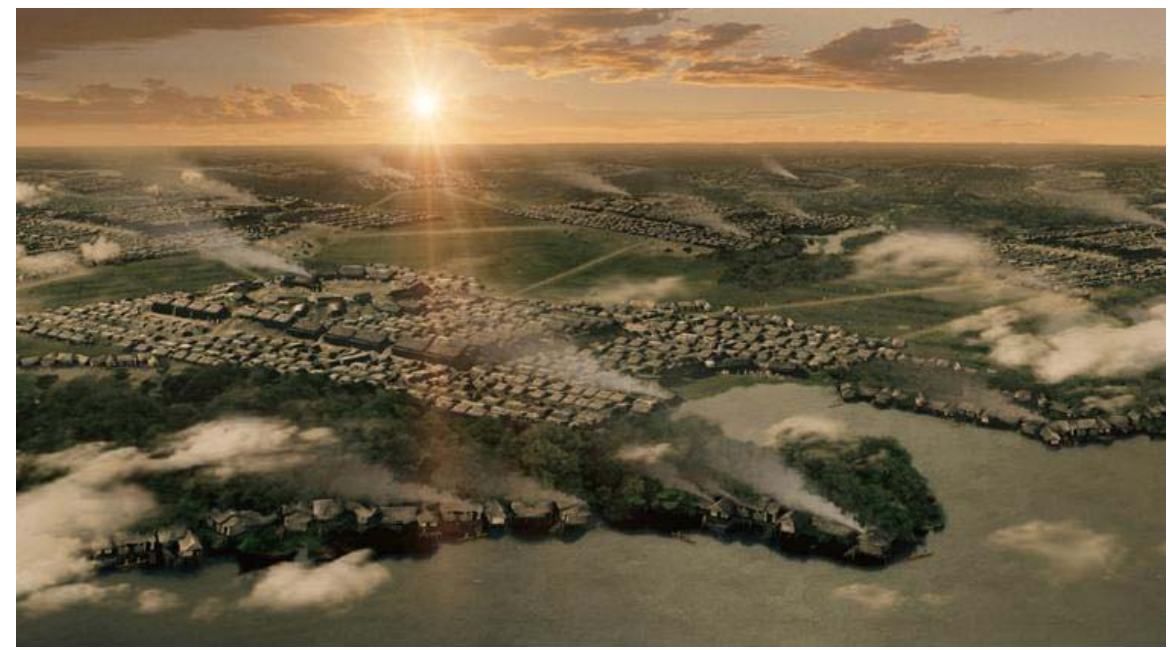

Figura 1.

Reconstrucción de la ciudad prehistórica de Açutuba, Amazonía Central.

Para evaluar la credibilidad de estas interpretaciones, los arqueólogos brasileños hicieron reconocimientos sistemáticos a lo largo de los principales afluentes del río Amazonas durante las últimas tres décadas bajo el Programa Nacional de Investigaciones Arqueológicas en la Cuenca Amazónica (PRONAPABA). La existencia de datos comparables de zonas muy separadas, cronologías relativas detalladas y numerosos fechados radiocarbónicos permite identificar comunidades prehistóricas, reconstruir su comportamiento residencial y social y correlacionar sus distribuciones temporales y espaciales con rasgos ambientales y fluctuaciones climáticas. Estos datos indican que aldeas pequeñas igualitarias, semejantes a las comunidades indígenas tradicionales sobrevivientes, se desarrollaron al menos cuatro mil años antes del contacto europeo y que constituyen una adaptación sostenible a las limitaciones ambientales inherentes y fluctuantes.

La reconstrucción de la prehistoria amazónica es impedida por la escasez de la evidencia arqueológica. El carácter perecible de la cultura material limita los 
vestigios a la cerámica y su disposición en los sitios de habitación. La inspección de secuencias seriadas de regiones muy separadas llamó la atención a características de las frecuencias y tendencias de los tipos decorados y no decorados atribuibles a la influencia de la deriva evolutiva. Esta correlación indica que las fases identificadas por secuencias seriadas representan comunidades prehistóricas endógamas y las seriaciones proveen información sobre su comportamiento habitacional y social.

\section{Metodología}

La construcción de secuencias seriadas depende de la existencia de muestras de tiestos no seleccionados de la superficie y de excavaciones estratigráficas y su clasificación por criterios uniformes. Como los tiestos sin decoración normalmente constituyen entre el 90 y $100 \%$ de una muestra no seleccionada, representan una fuente significativa de información (Fig. 2). Se clasifican los tiestos sin decoración en base al antiplástico y los decorados en base a la técnica de decoración. La frecuencia relativa de cada tipo en cada nivel se calcula en base al total del nivel, los resultados son dibujados en orden estratigráfico para cada corte. Niveles con tendencias y frecuencias relativas compatibles se intercalan para producir una secuencia seriada, que representa una cronología relativa. Los sitios incluidos identifican el territorio de una fase, que corresponde a una comunidad endógama.

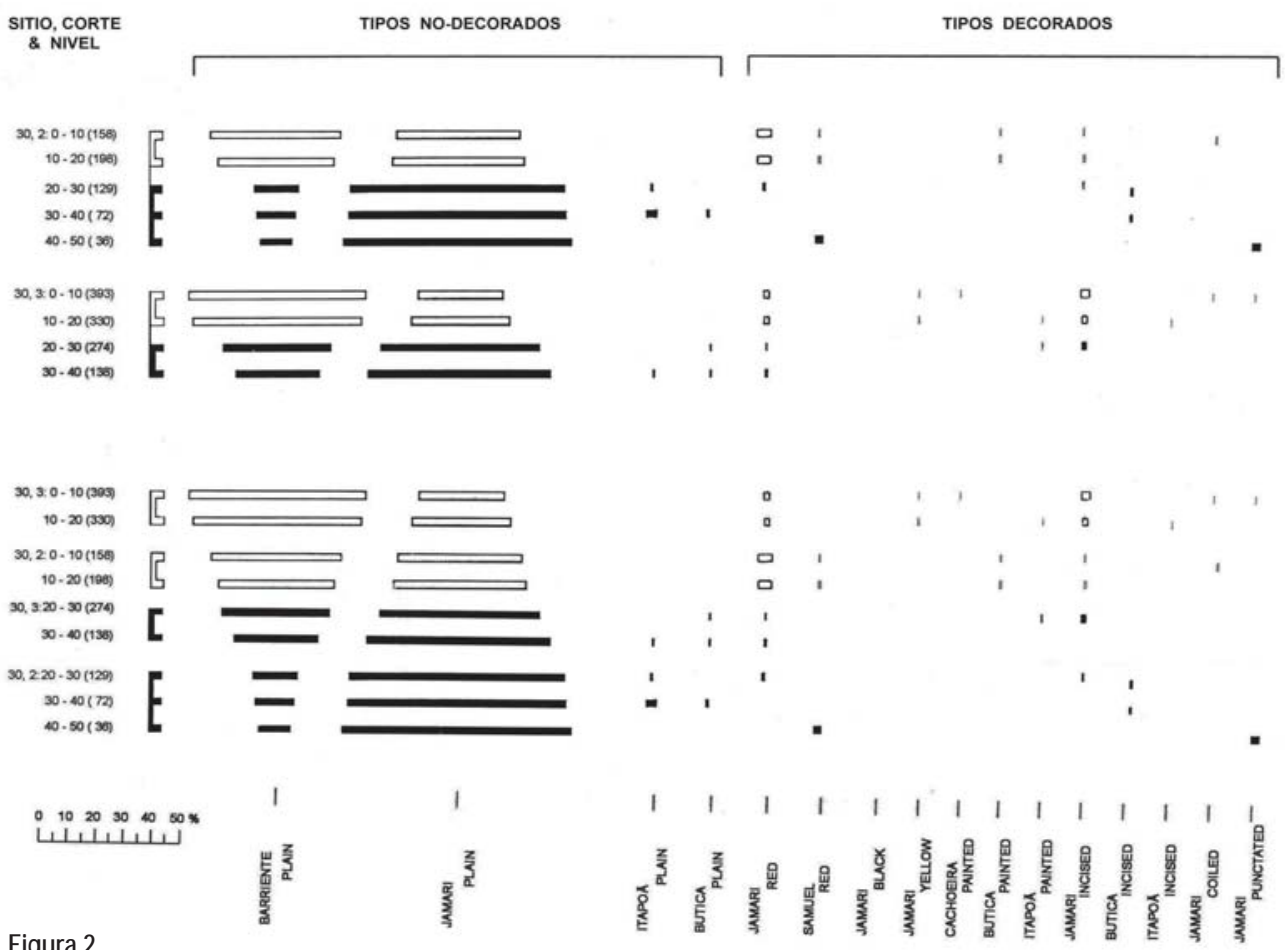

Figura 2.

Frecuencias relativas de tipos no-decorados y decorados en 2 cortes, y su intercaiación. 


\section{Evidencia Arqueológica}

Las regiones con la evidencia arqueológica más detallada son el Tocantins, el tributario oriental del lado derecho del bajo Amazonas, y el Jamarí, un tributario del lado derecho del alto Madeira en el suroeste.

\section{Río Tocantins}

El reconocimiento del Tocantins se limitó al sector sujeto a inundación por la construcción del embalse hidroeléctrico en Tucuruí. Las diferencias en las tendencias y frecuencias relativas de los tipos de cerámica en recolecciones de superficie y cortes estratigráficos en 35 sitios identificaron cinco fases con territorios contiguos que se correlacionan con sectores del río con diferentes recursos acuáticos de subsistencia (Fig.3).

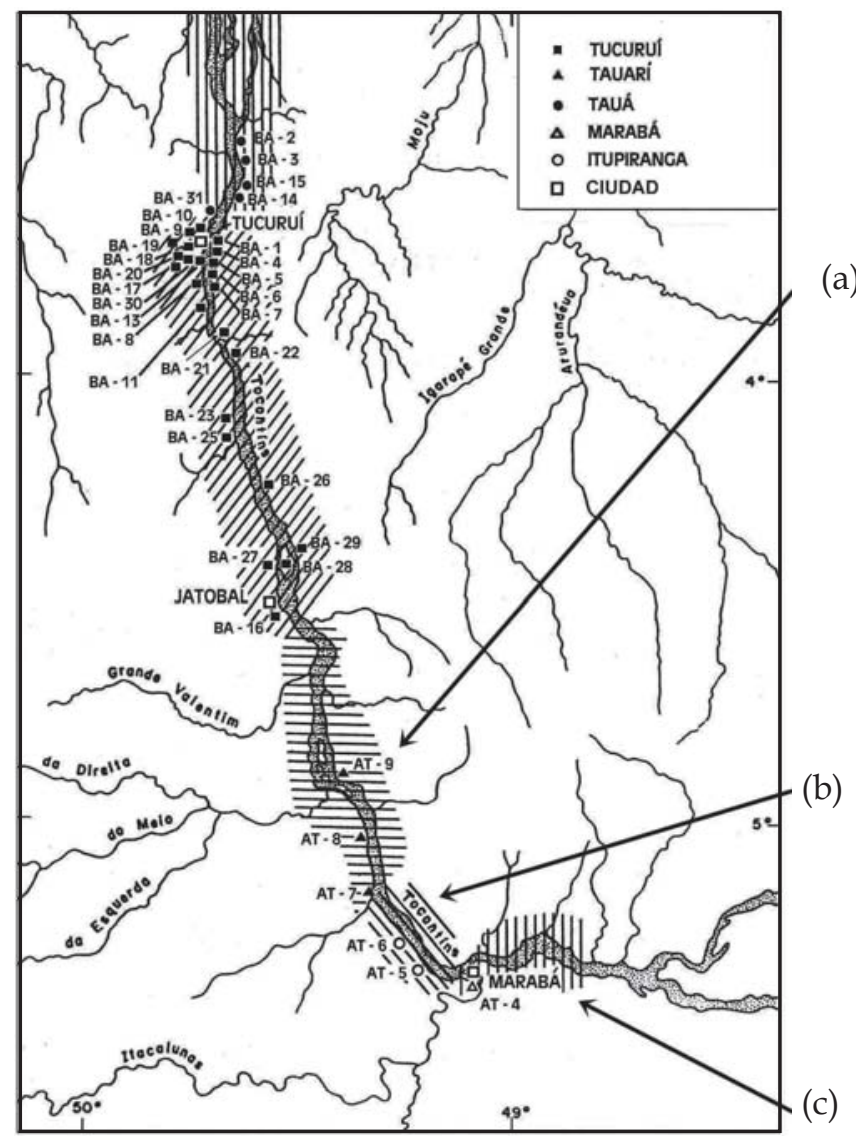

Figura 3.

Seriaciones identificando 3 fases contemporáneas en el bajo Tocantins y sus territorios. 


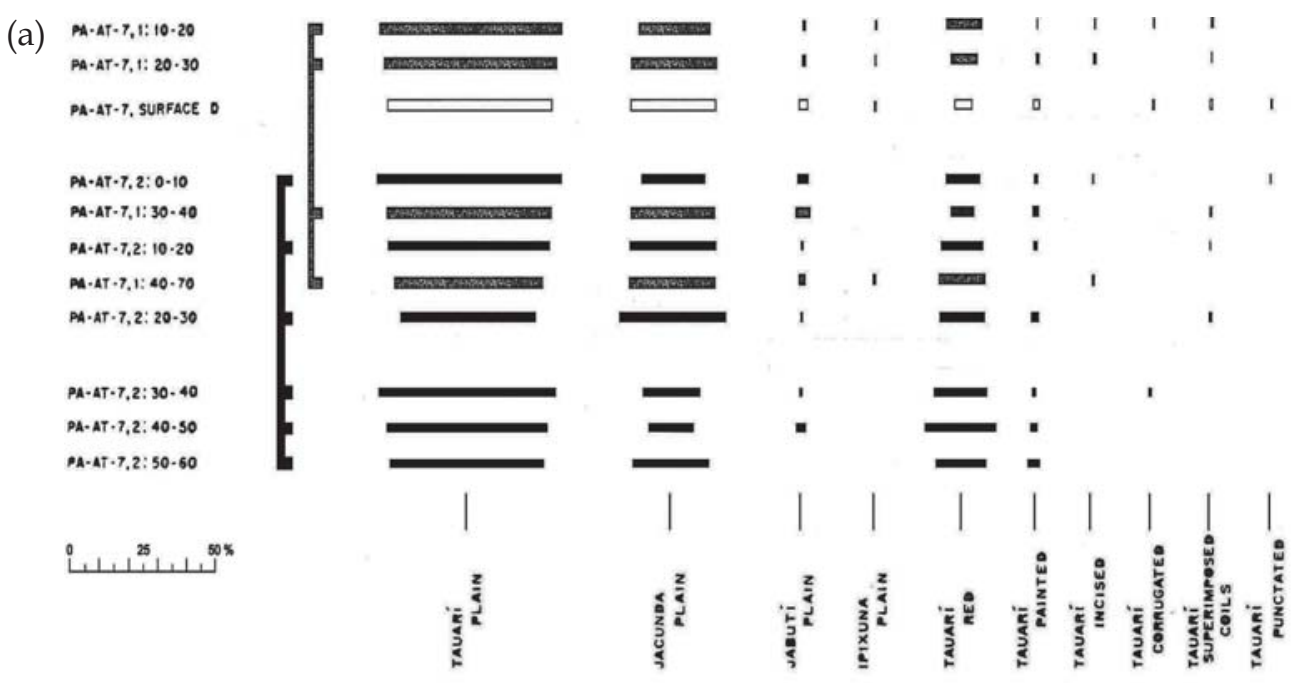

(b) PA-AT-6: $0.10 \mathrm{cM}$
PA-AT-6: $10-20$
PA-AT-5, SURFACE
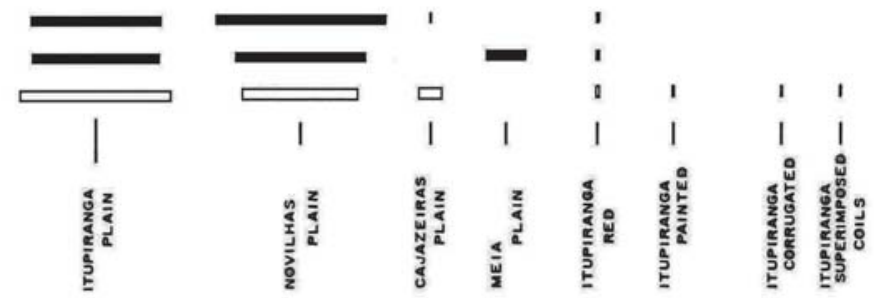

(c) 25.
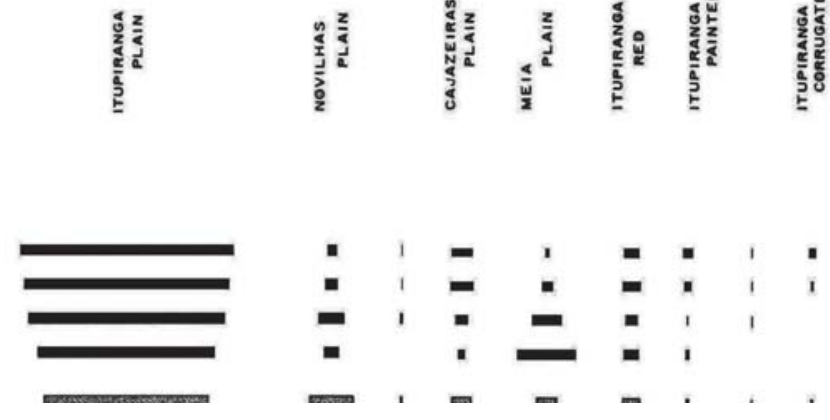

La inspección de la secuencia seriada de la Fase Tucuruí reveló una fluctuación errática en la frecuencia relativa de algunos de los tipos decorados que no se puede explicar por error del muestreo (Fig. 4). Separando las muestras en base de la presencia de más o menos $5 \%$ de Tucuruí Pintado produjo dos seriaciones contemporáneas y la inspección de la localización de los sitios reveló que solo dos fueron ocupados y reocupados exclusivamente por una de las sub-fases. La diferencia cuantitativa en la presencia de pintura se explica por deriva evolutiva e implica el aislamiento de las mujeres por residencia matrilocal. 


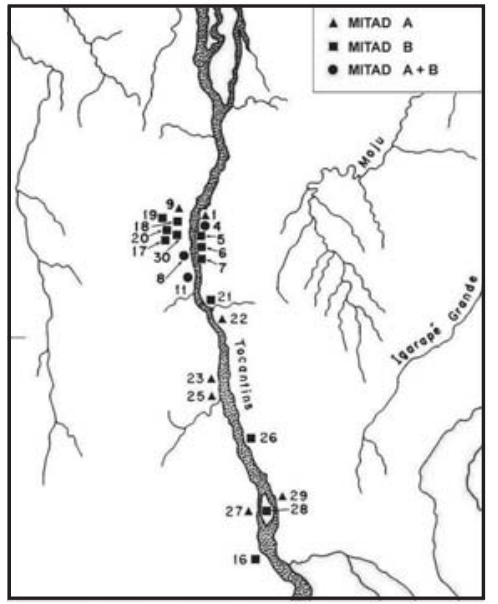

Figura 4.

Seriaciones seriadas de las mitades de la fase Tucuruí y distribución de los sitios (río Tocantins).
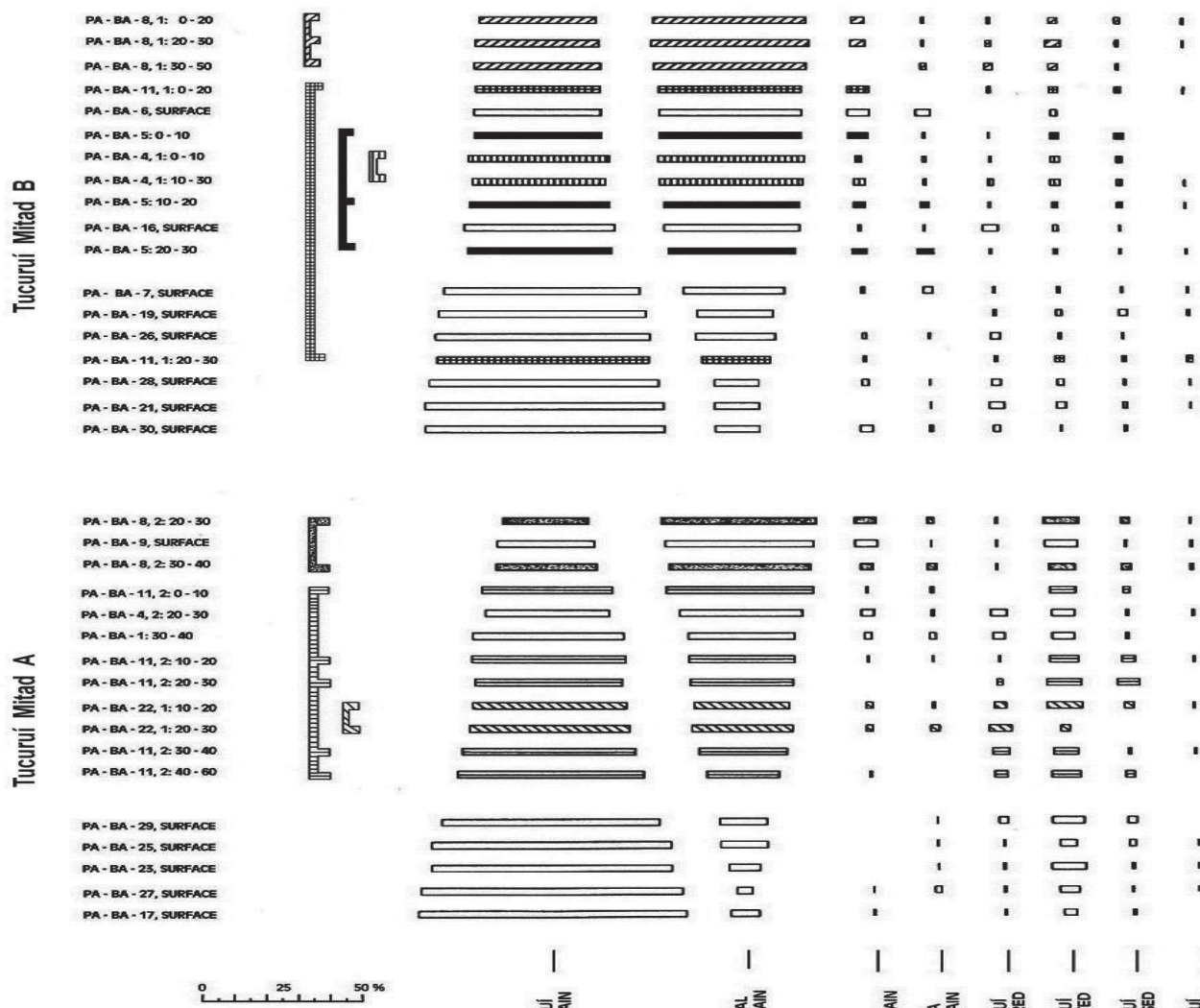

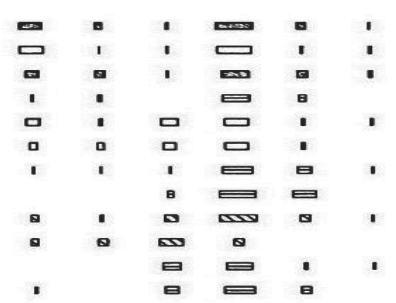
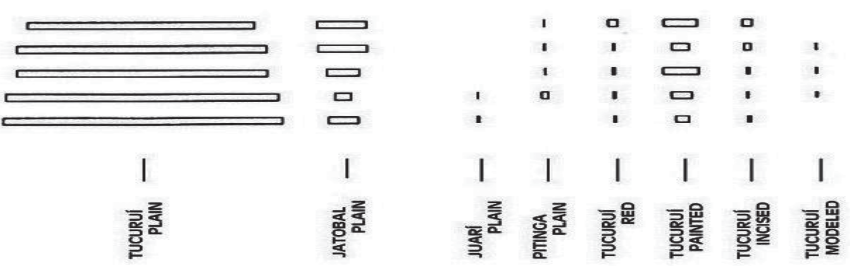


\section{Río Jamarí}

La prospección intensiva del río Jamarí en la Amazonía occidental identificó 121 sitios en una distancia de $260 \mathrm{~km}$. arriba del primer rápido, incluyendo 89 sitios cerámicos prehistóricos y 8 campamentos con cerámica. Muestras de cerámica no seleccionada existen de 42 recolecciones de superficie, y entre una y nueve excavaciones estratigráficas en 22 sitios. La cronología relativa se complementa con 137 fechados de carbono-14 (Miller et al, 1992).

\section{La ocupación precerámica}

Los artefactos líticos identifican tres fases precerámicas entre 8200 y 2500 AP. Los artefactos líticos de las dos fases más antiguas se encuentran en suelo que no muestra cambio del color, significando campamentos breves de cazadores/ recolectores.

La siguiente Fase Massangana se identificó por depósitos de terra preta con artefactos líticos debajo de ocupaciones cerámicas en 20 sitios distribuidos por toda el área prospectada (Fig. 5). Treinta fechados de carbono-14 se extienden desde 4880 « 60 hasta $2500 » 90 \mathrm{AP}$. La apariencia de hachas y morteros entre los artefactos líticos indica un cambio en la subsistencia y la existencia de terra preta implica la adopción del comportamiento habitacional semi-sedentario asociado con la agricultura de roza y quema antes de la adopción de la cerámica.

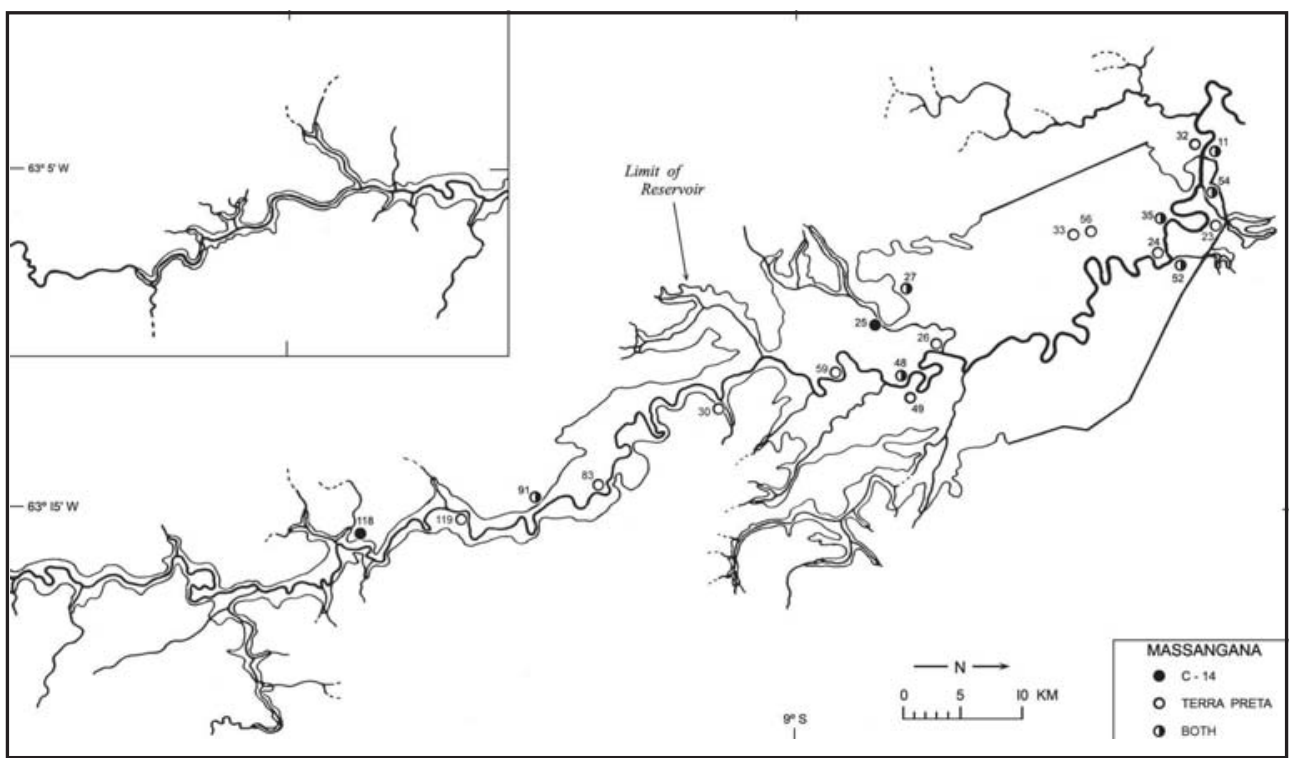

Figura 5.

Sitios de la fase precerámica Masangana, en el río Jamarí 
Fases Cerámicas. La cerámica fue introducida cerca 2400 AP y las secuencias seriadas identifican cinco fases de la misma tradición con distribuciones territoriales y duraciones temporales diferentes. Como en el río Tocantins, todas se dividen en dos mitades matrilocales que ocupaban sitios diferentes.

La Fase Urucurí más temprana, se ha identificado en 18 sitios distribuidos en toda la región prospeccionada (Fig.6). El complejo cerámico consiste en dos tipos no decorados y cinco tipos decorados. La presencia o ausencia de incisión divide la secuencia seriada en dos mitades que ocuparon sitios diferentes.

Cerca de 1500 AP, la Fase Urucurí fue suplantada por la Fase Jamarí en el norte (Fig.7). Los dos tipos principales no decorados tienen antiplástico de arena o cariapé, pero el cauixí existe en menor frecuencia o solo o combinado con cariapé. La decoración más común es baño rojo. Engobe rojo, pintura, incisión fina y ancha, rollos no apagados y excisión ocurren erráticamente en menor frecuencia durante toda la secuencia. Las diferencias en las frecuencias relativas del baño rojo y engobe rojo identifican dos mitades matrilocales que ocuparon sitios diferentes.

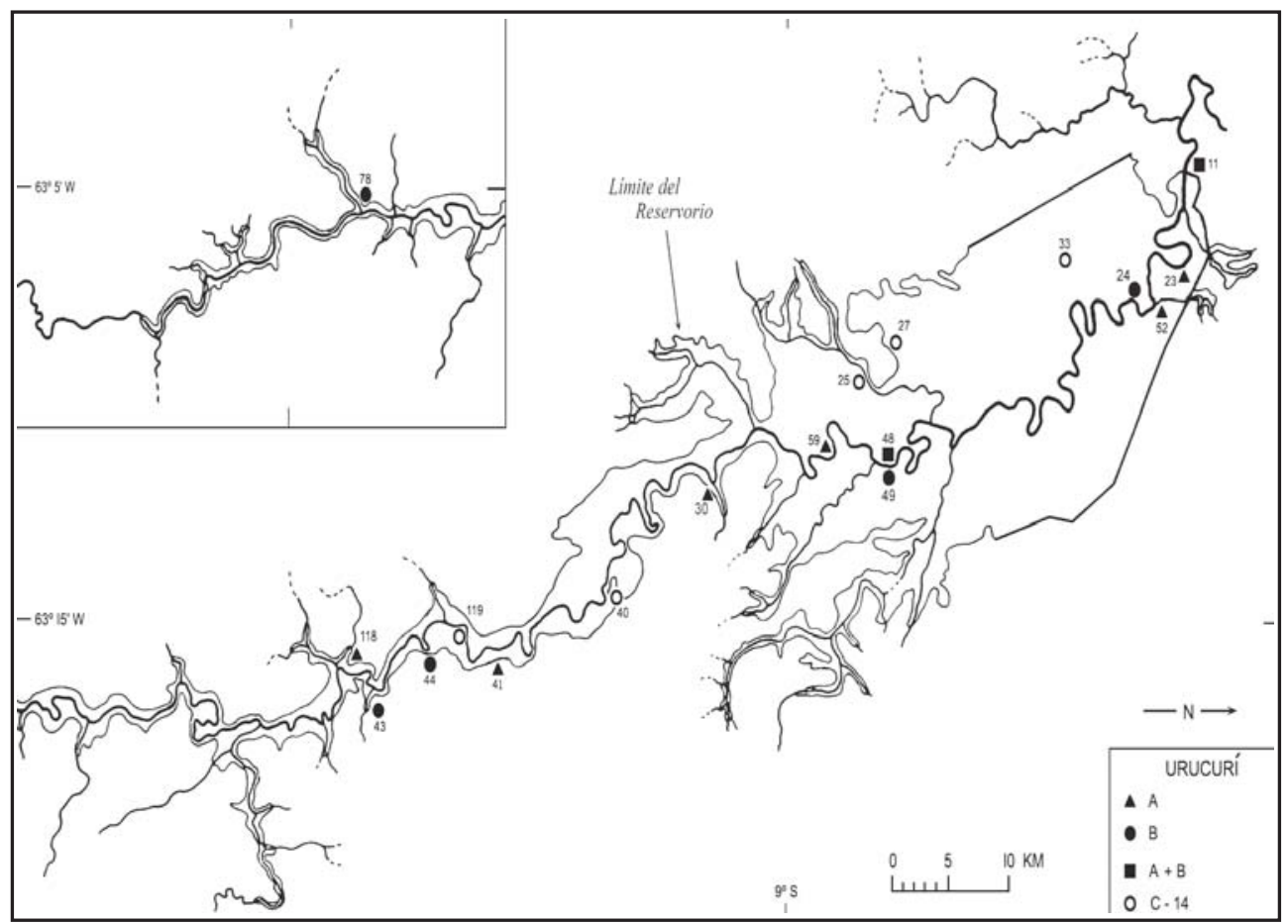

Figura 6.

Sitios y seriaciones de las mitades A y B de la fase Urucurí en el río Jamarí. 


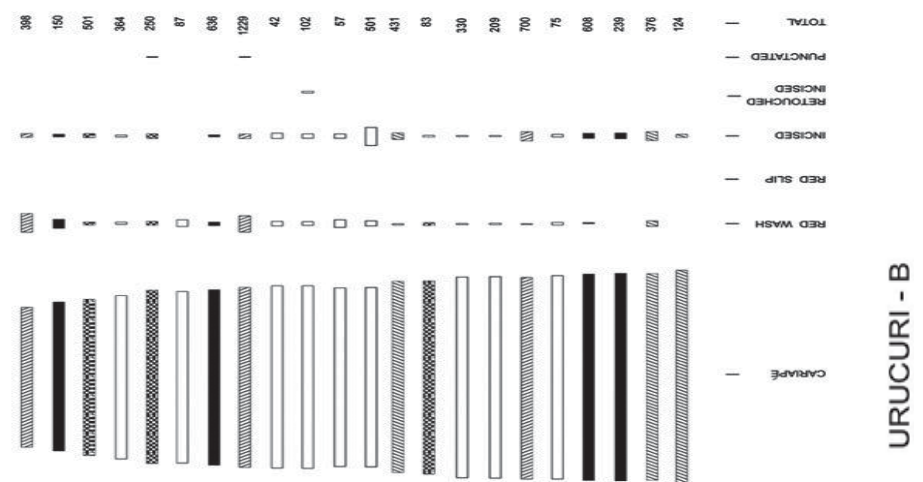

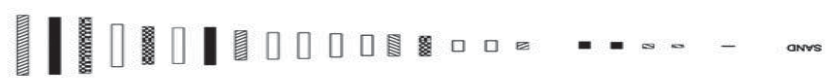
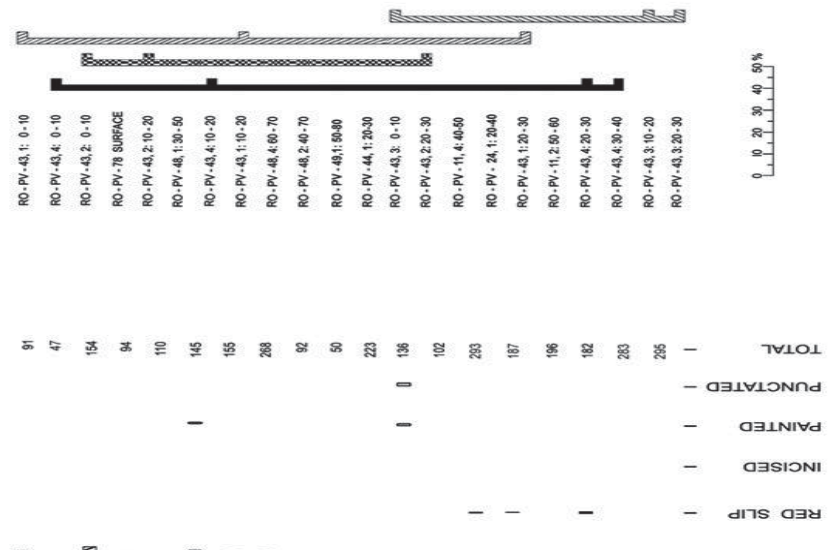

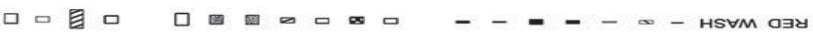

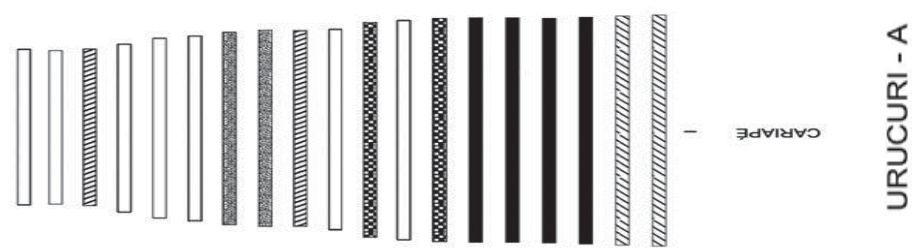

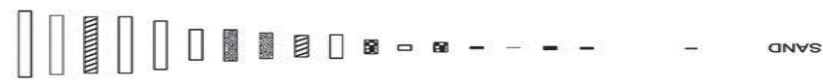

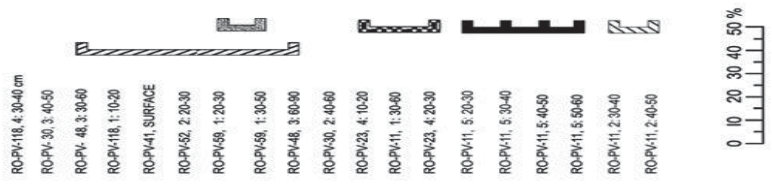



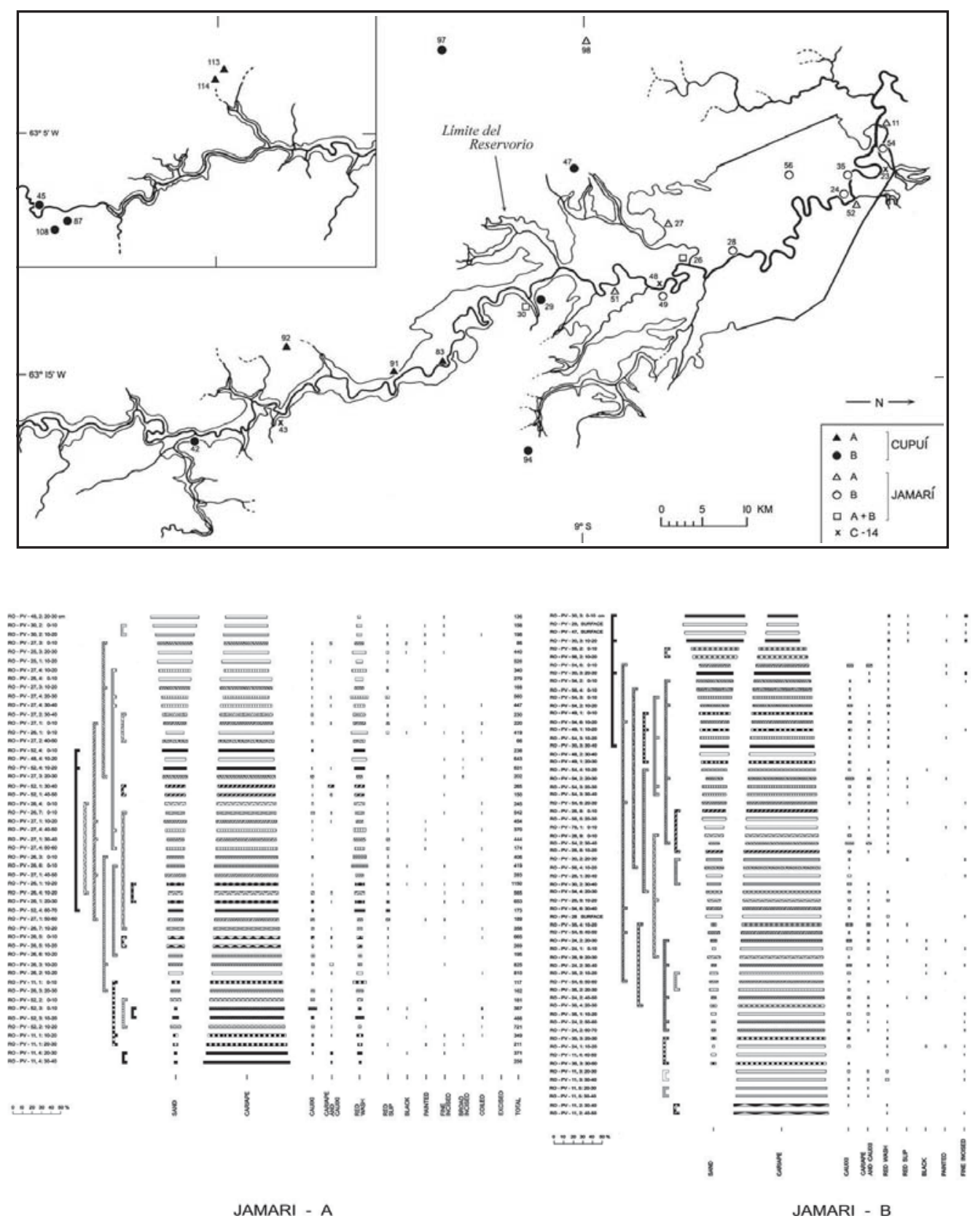

Figura 7.

Sitios de las fases Jamarí y Cupuí, y las seriaciones de las mitades. 
Aproximadamente al mismo tiempo, la Fase Urucurí fue suplantada por la Fase Cupuí en la parte sur del territorio (Fig. 8). Los tipos principales no decorados tienen antiplástico de arena o cariapé, pero las tendencias y frecuencias relativas son opuestas a las de la Fase Jamarí y la escasa decoración se limita al baño rojo, incisión y escovado. La presencia o ausencia de antiplástico de cauixí o cauixítcariapé identifica dos mitades, que ocuparan sitios diferentes. Como en el Tocantins, la frontera entre las dos fases correlaciona con un cambio en la productividad del río.

Aproximadamente a los 700 AP, la Fase Jamarí fue suplantada por la Fase Matapí en el norte y la Fase Topazio en el sur (Fig. 9). Las tendencias y frecuencias relativas de los dos tipos no decorados son semejantes a las fases anteriores, pero existe poca o ninguna decoración. Diferencias en las frecuencias relativas de los tipos no decorados identifican dos mitades en ambas fases, que ocuparon sitios diferentes.

Reocupación. Una comparación de las ubicaciones de los 49 sitios de las fases cerámicas revela que solamente 6 fueron ocupados durante tres fases seguidas, mientras que 26 fueron ocupados durante una sola fase (Fig. 10).

Durante cada fase, la mayoría de los sitios fueron ocupados y reocupados por la misma mitad. Algunos fueron ocupados por ambas mitades de la misma fase o reocupados durante una fase posterior y en ambos casos la nueva aldea típicamente se ubicó al lado, en lugar de sobre la basura anterior, aumentando la extensión de la terra preta. Por ejemplo, nueve excavaciones en RO-PV-26, un sitio de la Fase Jamarí, identifican ocupaciones de la Mitad A en los Cortes 1 hasta 7 y de la Mitad B en los Cortes 8 y 9 (Fig. 11). La semejanza de las frecuencias relativas en algunos niveles en la seriación de la Mitad A sugiere la presencia de dos o tres casas durante algunos episodios, mientras que las diferencias entre los niveles de la Mitad B sugieren discontinuidad de ocupación.

Una segregación espacial semejante ocurre en los sitios reocupados por fases diferentes. Seis excavaciones en RO-PV-54 indican que fue ocupado primero por la Mitad B de la Fase Jamarí en los Cortes 2, 3, 4 y 6 (Fig. 12). Una reocupación posterior por la Mitad B de la Fase Matapí, identificada en los Cortes 1 y 5, aumentó significativamente las dimensiones de la terra preta.

La frecuencia de reocupación en RO-PV-35 es comprobada por los fechados de cuatro cortes estratigráficos (Fig. 13). Fechados de niveles consecutivos de $10 \mathrm{~cm}$ difieren por 1500, 900 y 120 años, y fechados de la misma profundidad en excavaciones diferentes también difieren por cientos de años.

Esta evidencia niega las suposiciones que el tamaño de la terra preta corresponde al tamaño de la aldea, que la ocupación fue permanente y que todas las manchas son contemporáneas. 


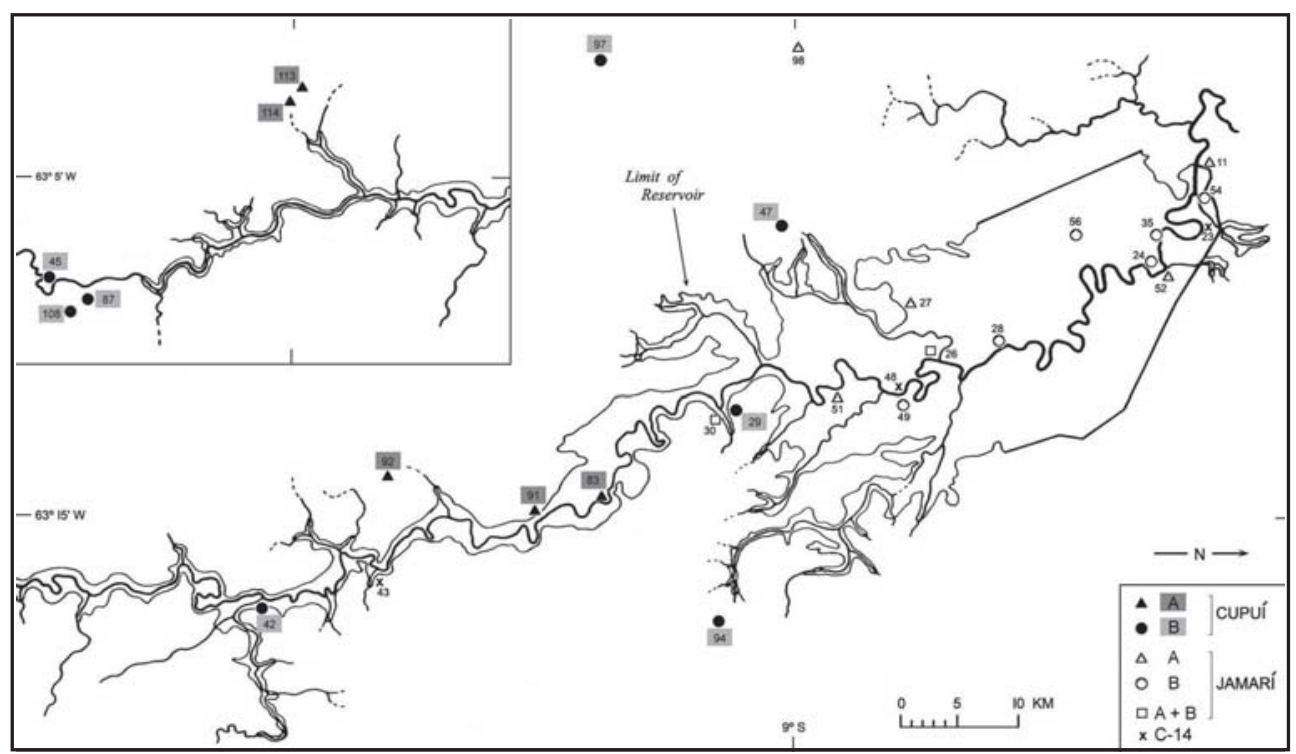

RO - PV - 92, SURFACE RO - PV - 83, 1: $0-10 \mathrm{~cm}$ RO - PV - 83, 1: 10 - 20 RO - PV - 91, 1: 20 - 30 RO - PV - 91, 1: $30-50$ RO - PV - 83, 1: 20 - 30 RO - PV - 114, 1:10 - 20 RO - PV - 83, 1: $30=50$ RO - PV - 113, 1:10 - 20

RO - PV - 47, SURFACE RO - PV - 29, SURFACE RO - PV - 45, SURFACE RO - PV - 108, SURFACE RO - PV - 97, SURFACE RO - PV - 42, SURFACE RO - PV - 87, SURFACE RO - PV - 94. SURFACE
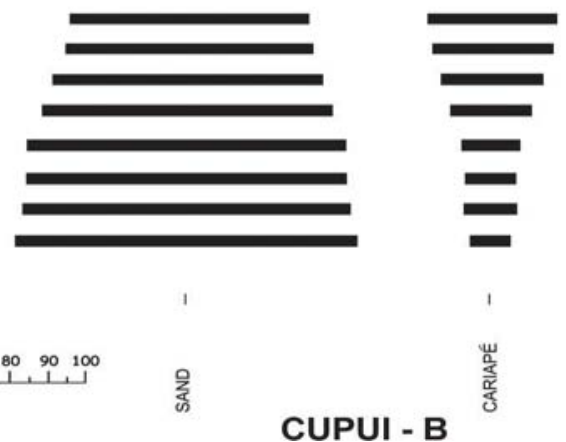

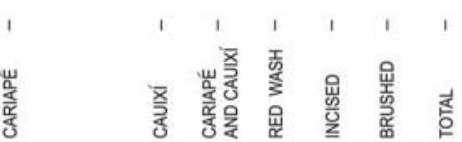

Figura 8.

Sitios de las fases Jamarí y Cupuí y las seriaciones de las mitades. 

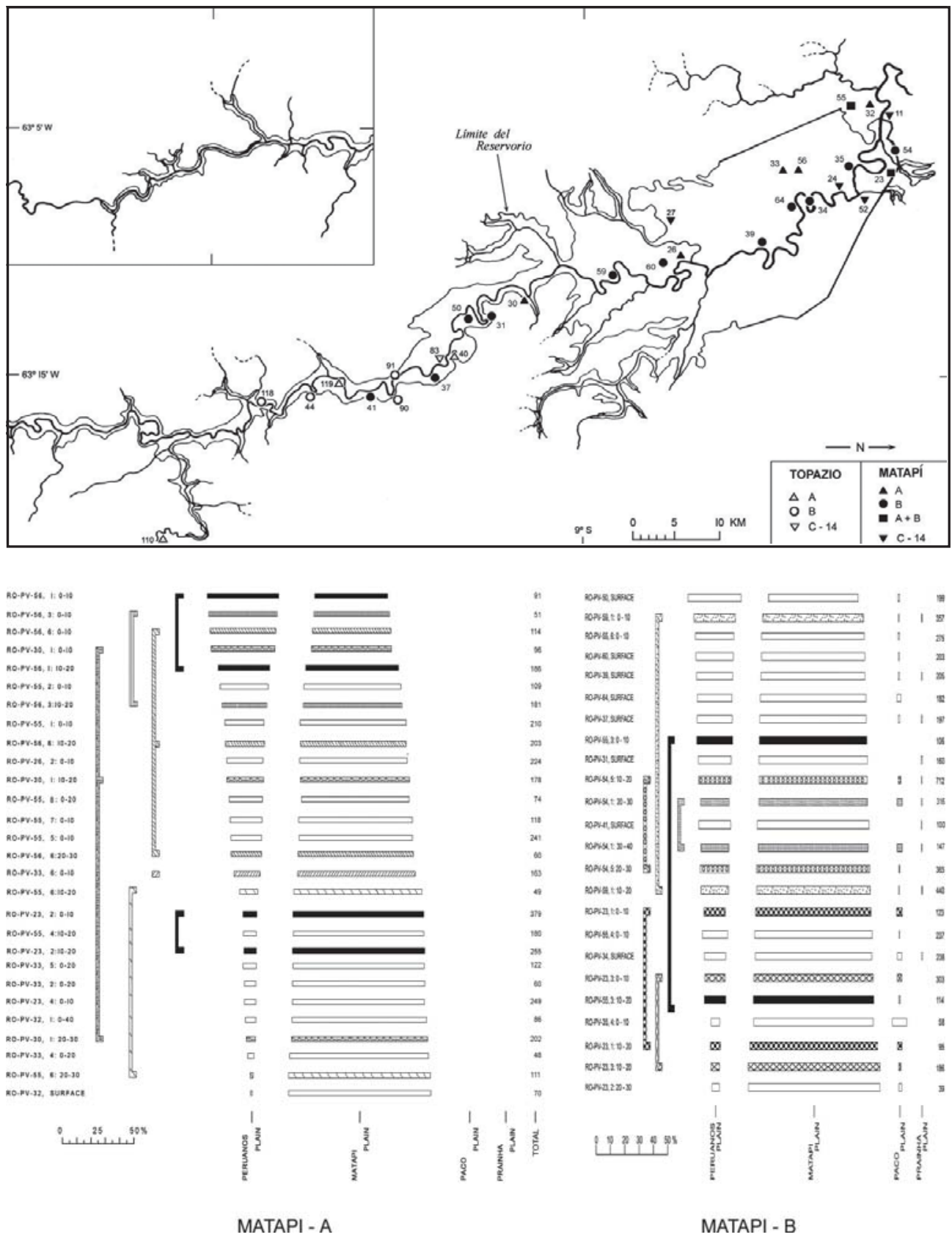

Figura 9.

Sitios de las fases Matapí y Topazio y las seriaciones de las mitades de la fase Matapí. 


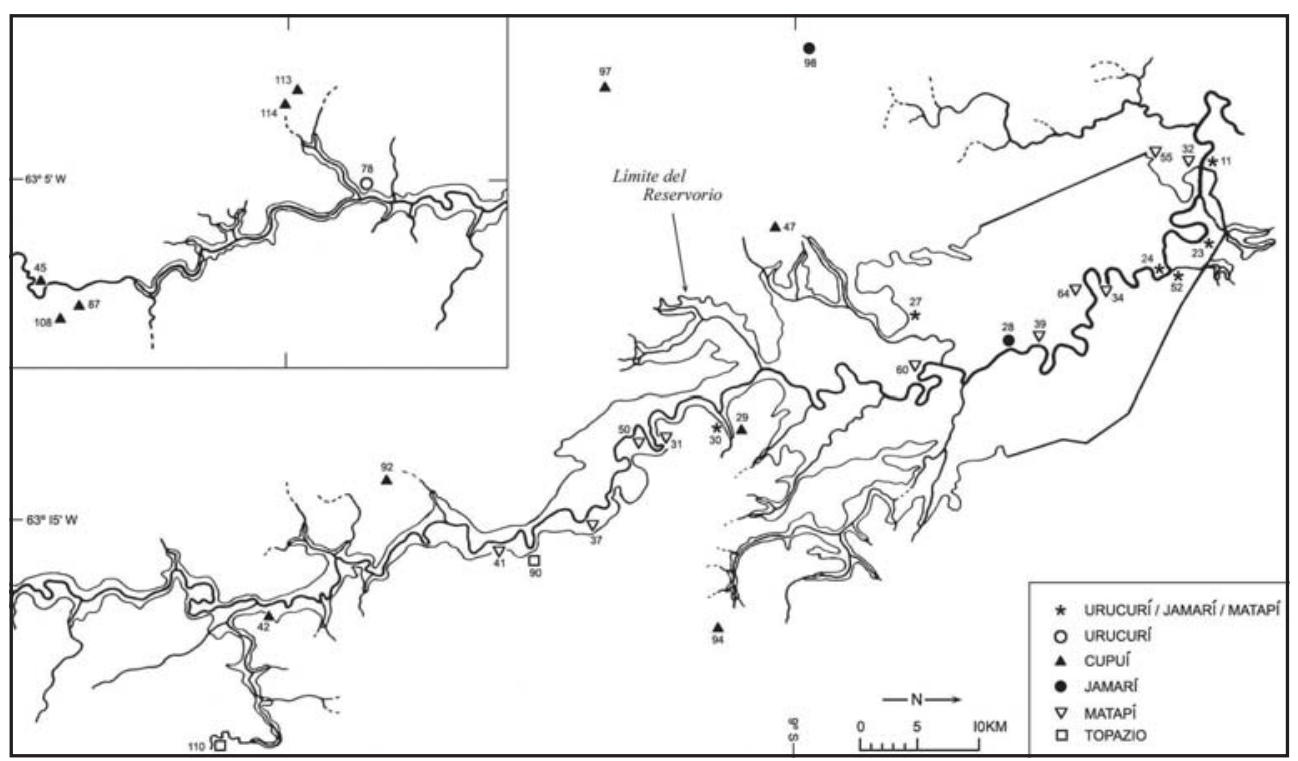

Figura 10.

Ubicaciones de los sitios reocupados durante 3 fases consecutivas y los sitios ocupados durante una sola fase.

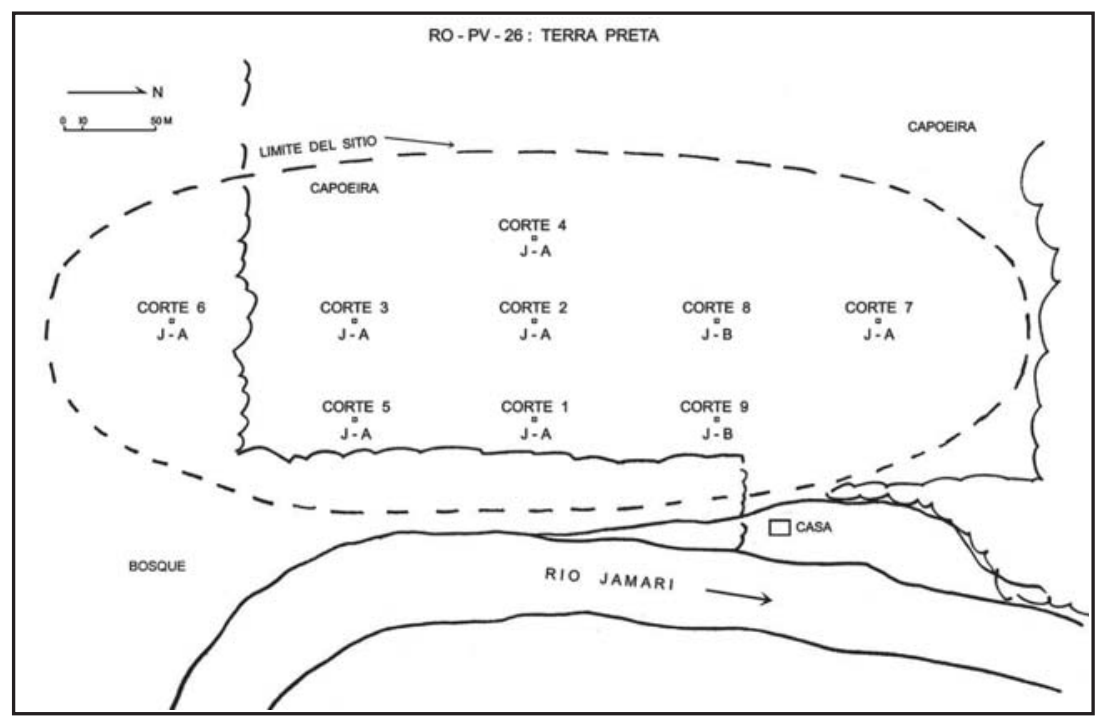

Figura 11.

Seriación de niveles en 9 cortes, identificando ocupaciones de las mitades A y B de la fase Jamarí. 
๙ิ

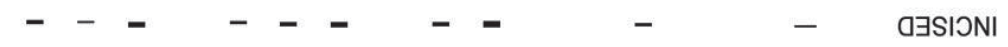

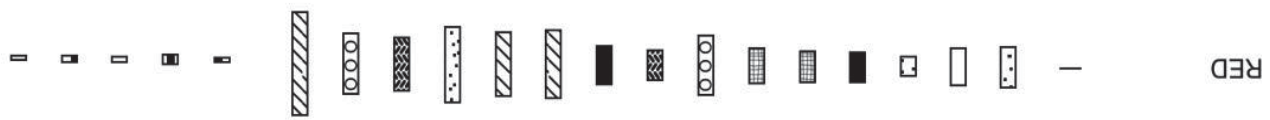

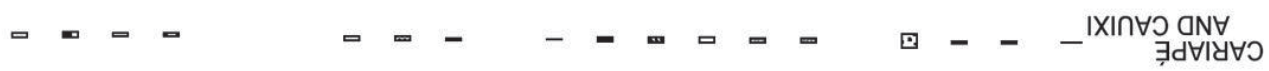

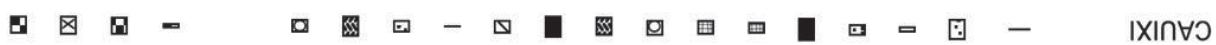
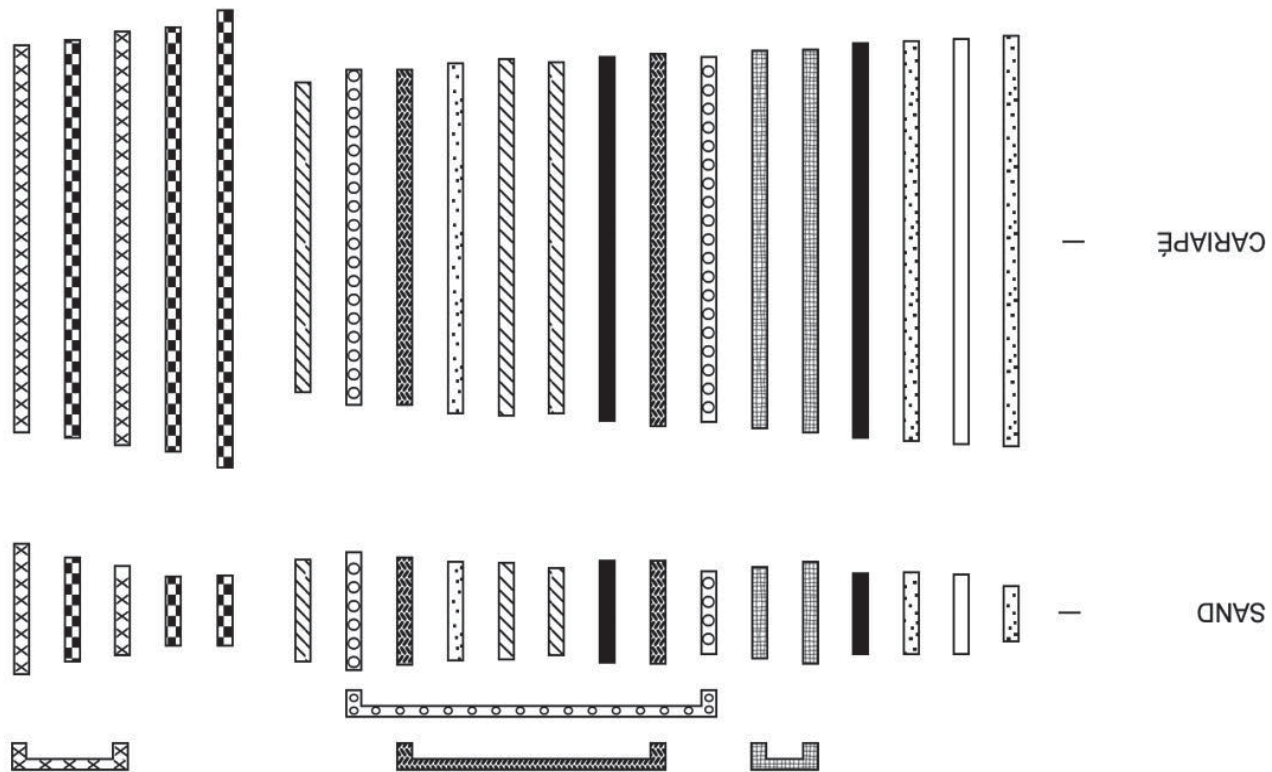

ming

Bmm

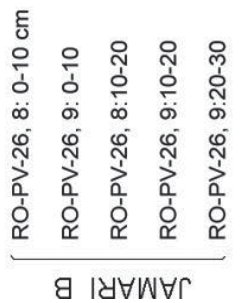
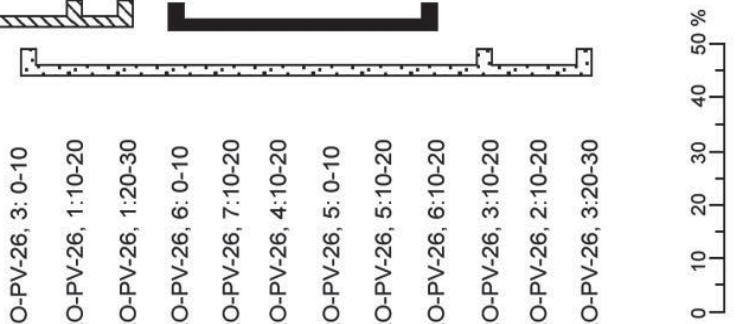

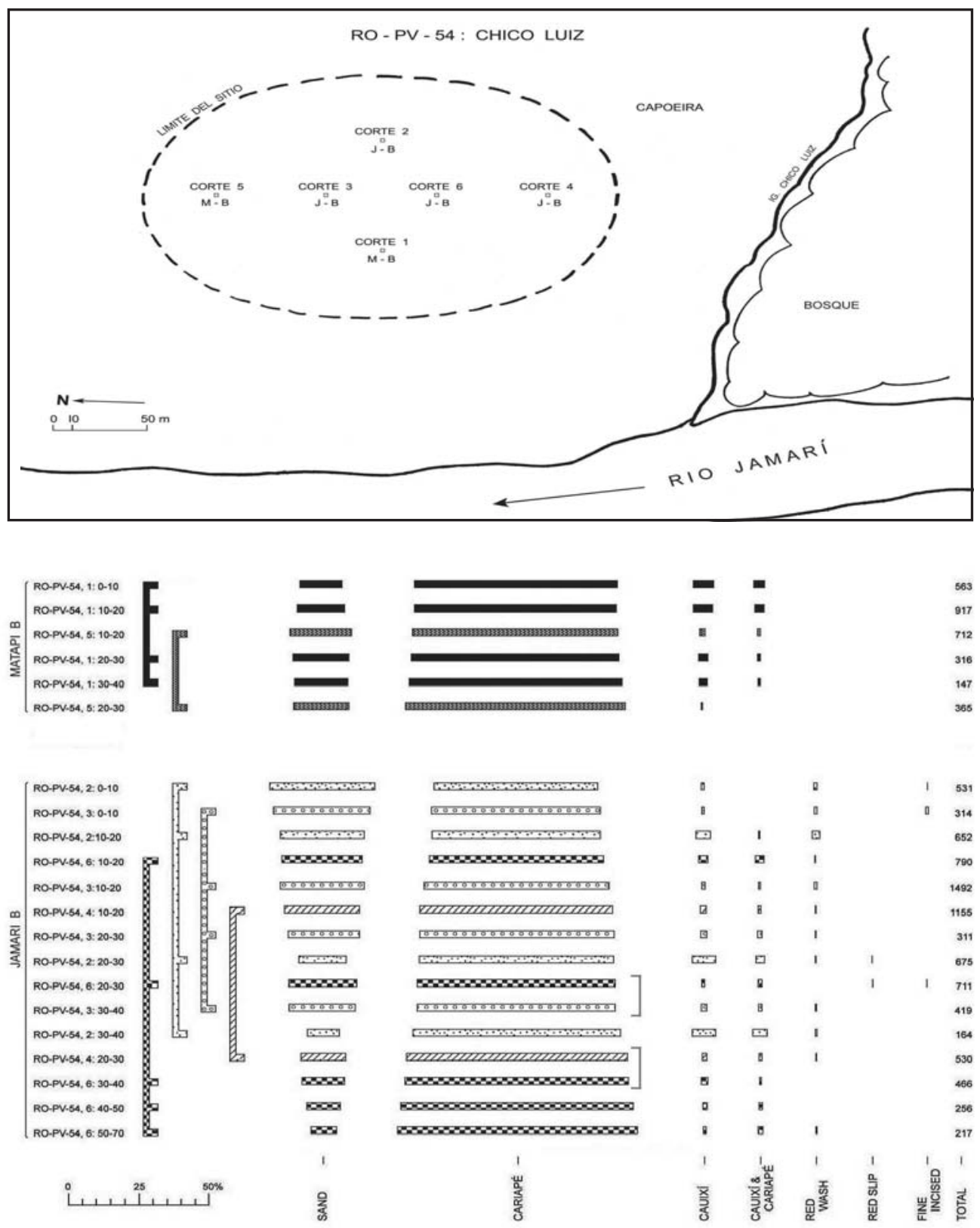

Figura 12.

Seriación de los niveles en seis cortes del sitio RO-PV-54 identificando ocupaciones de la fase Jamarí (cortes 2,3,4 y 6) y de la fase Matapí (cortes 1 y 5). 


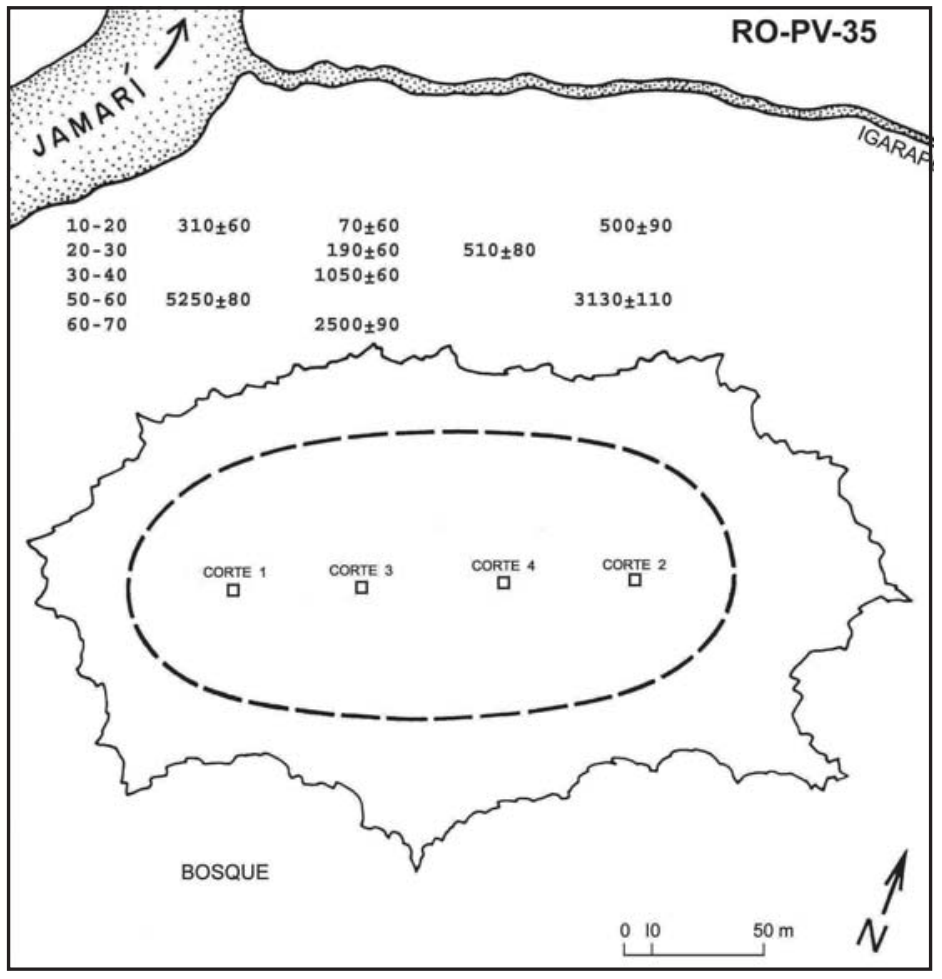

Figura 13.

Discrepancias en los fechados radiocarbónicos en niveles consecutivos y de la misma profundidad.

Una segregación espacial semejante ocurre en los sitios reocupados por fases diferentes. Seis excavaciones en RO-PV-54 indican que fue ocupado primero por la Mitad B de la Fase Jamarí en los Cortes 2, 3, 4 y 6 (Fig. 12). Una reocupación posterior por la Mitad B de la Fase Matapí, identificada en los Cortes 1 y 5, aumentó significativamente las dimensiones de la terra preta.

La frecuencia de reocupación en RO-PV-35 es comprobada por los fechados de cuatro cortes estratigráficos (Fig. 13). Fechados de niveles consecutivos de $10 \mathrm{~cm}$ difieren por 1500, 900 y 120 años, y fechados de la misma profundidad en excavaciones diferentes también difieren por cientos de años.

Esta evidencia niega las suposiciones que el tamaño de la terra preta corresponde al tamaño de la aldea, que la ocupación fue permanente y que todas las manchas son contemporáneas.

\section{Analogías etnográficas}

Si las reconstrucciones del comportamiento residencial y social prehistórico son válidas, deben mantenerse entre comunidades indígenas que conservan sus 
modos de vida tradicionales. Estos incluyen: 1) territorios contiguos ocupados por comunidades endógamas; 2) cambio periódico de la ubicación de la aldea con prioridad de reocupación de sus sitios anteriores; 3) residencia matrilocal y 4) evitación de los sitios de grupos anteriores.

Territorios endógamos. Un ejemplo de territorios contiguos bisectados por ríos y ocupados por comunidades endógamas, se ha registrado entre los Akawaio de la Guyana (Fig. 14). El aislamiento entre las comunidades se refleja en la existencia de pequeñas diferencias lingüísticas y culturales por influencia de la deriva evolutiva.

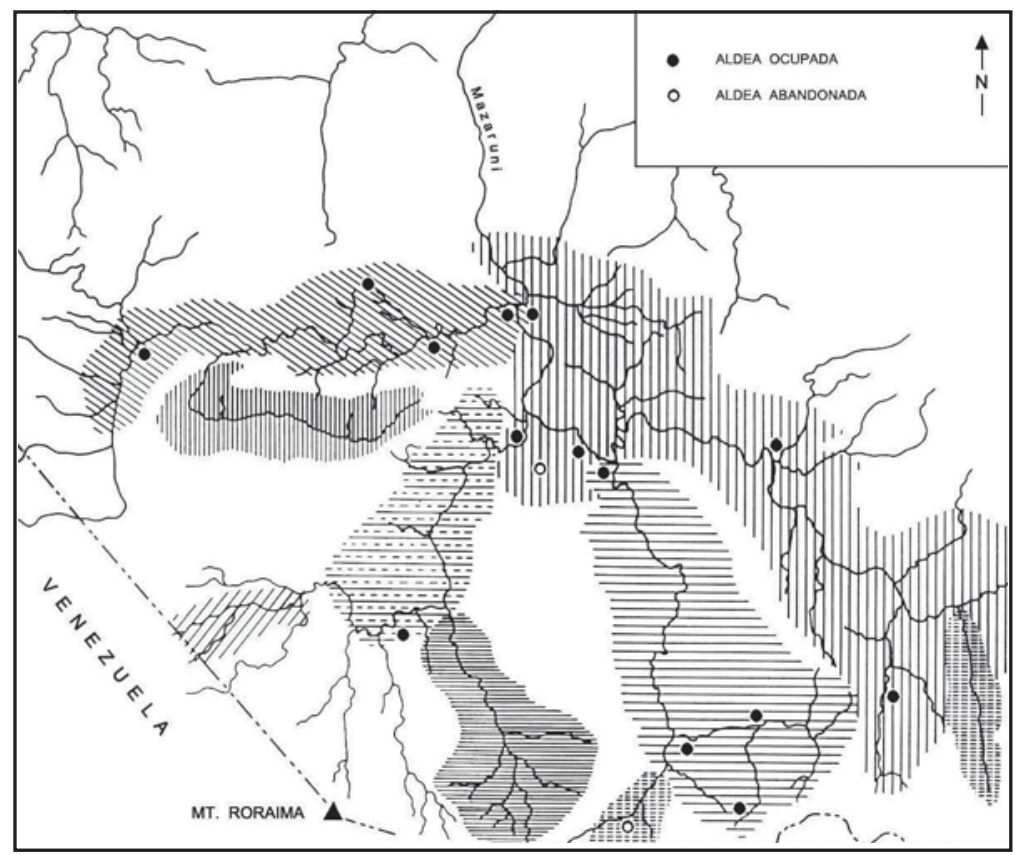

Figura 14.

Akawaio: territorio de las comunidades endógamas.

Traslado y Reocupación de Aldeas. Las aldeas típicas amazónicas consisten en una sola casa comunal, circular o extendida, que cambia de lugar cada 10 años aproximadamente por varias razones que incluyen el deterioro de la casa, el agotamiento de las presas o de la tierra de cultivo y la muerte de un ocupante (Fig. 15).

La reocupación de sus sitios anteriores se ha reportado entre los Kalapalo, Tapirapé, Cubeo, Akawaio , Piaroa , Yanomami , Waiãpi y Trumai. Los Siona-Secoya siguen un circuito durante unos 50 años y los Cubeo reocupan sitios anteriores después de 10-15 años. 


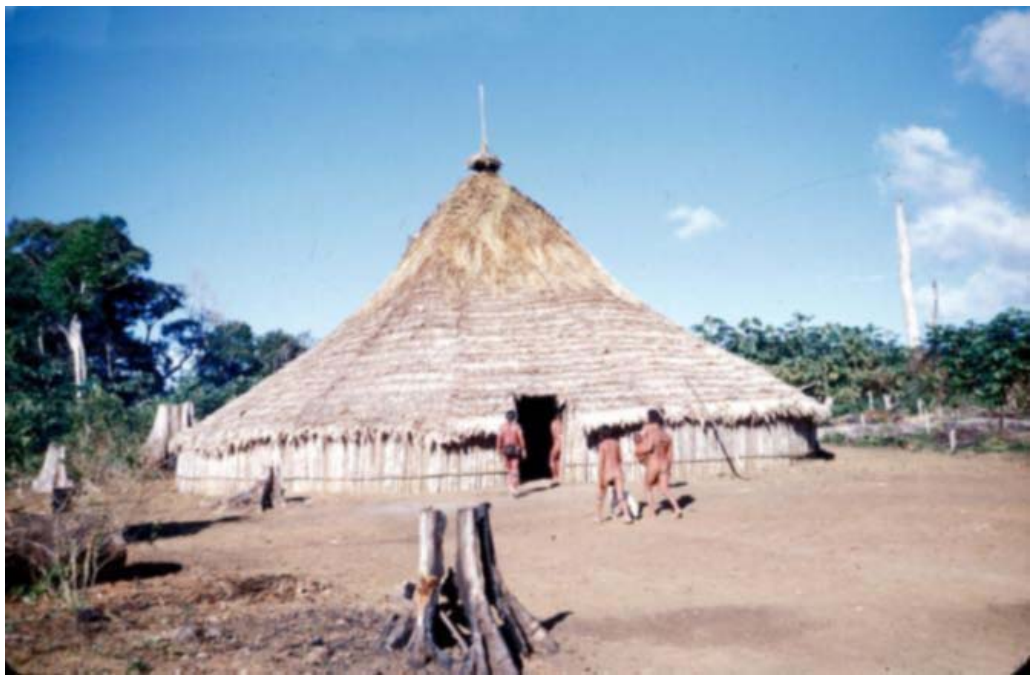

Figura 15.

Casa comunal Wai-Wai en la Guyana.

Residencia matrilocal. La residencia matrilocal es característica a través de la terra firme y el desarrollo de variaciones menores en la producción de la cerámica, como consecuencia del aislamiento entre las mujeres y la deriva evolutiva observada repetidamente. Entre los Shipibo, existe una interacción intensiva dentro de las residencias o grupos de casas matrilocales y poca entre ellos...». Como resultado de los patrones residenciales que aseguran que las niñas aprenden de sus madres y otras mujeres co-residentes, cada comunidad se hace el foco de un micro estilo permanente.

Entre los Bororo, la aldea está divida en dos mitades que muestran diferencias menores en decoración y formas de vasija (Wüst 1987-9).

Evitación de sitios de grupos anteriores. Aunque se ha prestado poca atención al hecho de evitar sitios antiguos por los grupos recientes, existen algunos ejemplos. Los Kalapalo no reocupan los sitios de habitación de los Trumai, que ellos reemplazaron en el alto Xingu. Entre los Tukano del oriente de Colombia, A los campos de cultivo o sitios de habitación antiguos, aunque no se conocen los dueños, constituyen una categoría muy especial del ecosistema....Los Tukano modernos tienen conciencia que fueron ocupados una vez por otra gente y los aproximan con admiración reverente».

Comportamiento de cacería. La conservación de otros rasgos generales del comportamiento residencial y social entre grupos contemporáneos sugiere que aspectos de la cacería también pueden ser una herencia del pasado. 
El territorio del poblado Yuquí en el oriente de Bolivia consiste en un núcleo con un diámetro de $10 \mathrm{~km}$ y una ampliación circundante de $5 \mathrm{~km}$., todo bisectado por el curso ondulado del Río Chimoré (Fig.16). Los 358 episodios de cacería durante 1983 excepto 2, y los 371 episodios durante 1988 excepto 19, se realizaron dentro del núcleo. Durante este período, una reducción en la caza preferida dentro del núcleo se compensó por un consumo incrementado de roedores. Esta disminución se atribuye a la intrusión de colonos dentro de la extensión, que aumentó significativamente la mortalidad de la fauna y eliminó la región como una fuente de reemplazo en el área nuclear.

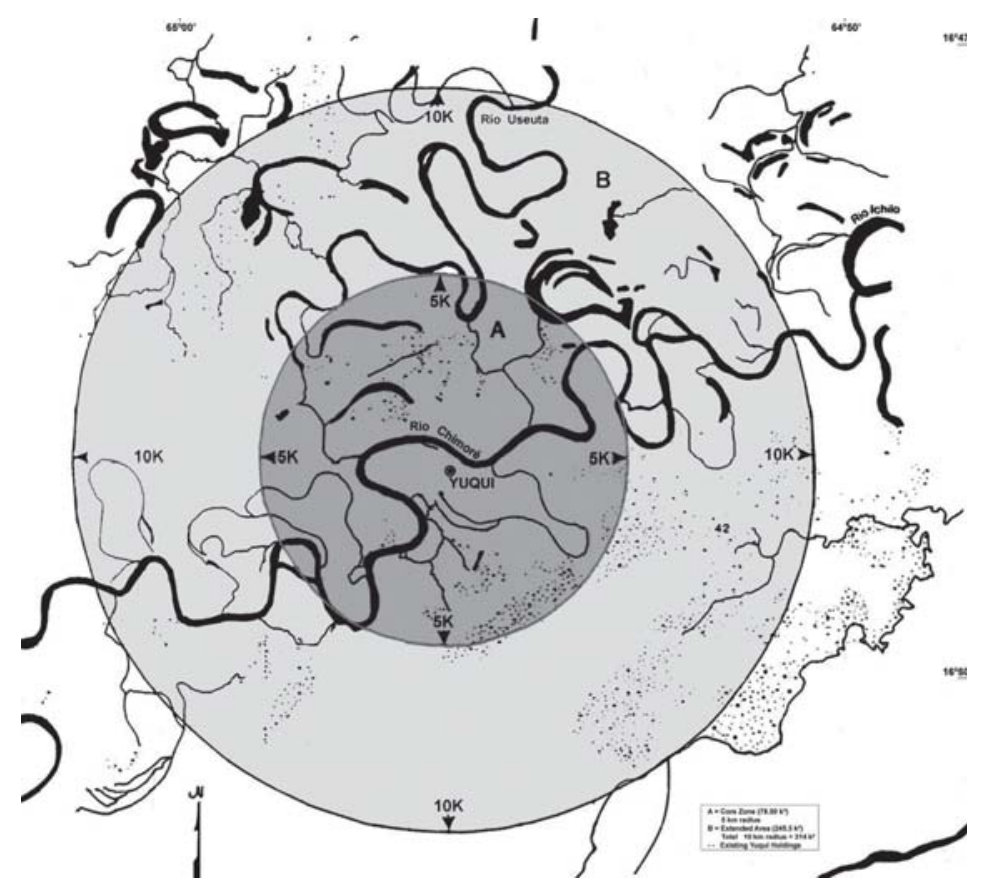

Figura 16.

Yuqui: Zona de cacería 1983-1988

Aunque la distribución de los sitios ocupados durante la Fase Jamarí da la impresión que el territorio fue sub-poblado, la proyección solamente del área nuclear con un diámetro de $10 \mathrm{~km}$ produce una superposición entre la mayoría de los sitios (Fig. 17). Como una explotación sostenible hubiera dependido del acceso a la región circundante para la rotación de zonas de cacería y el reemplazo natural del área nuclear, es obvio que todos los sitios no pudieron haber sido ocupados simultáneamente. 


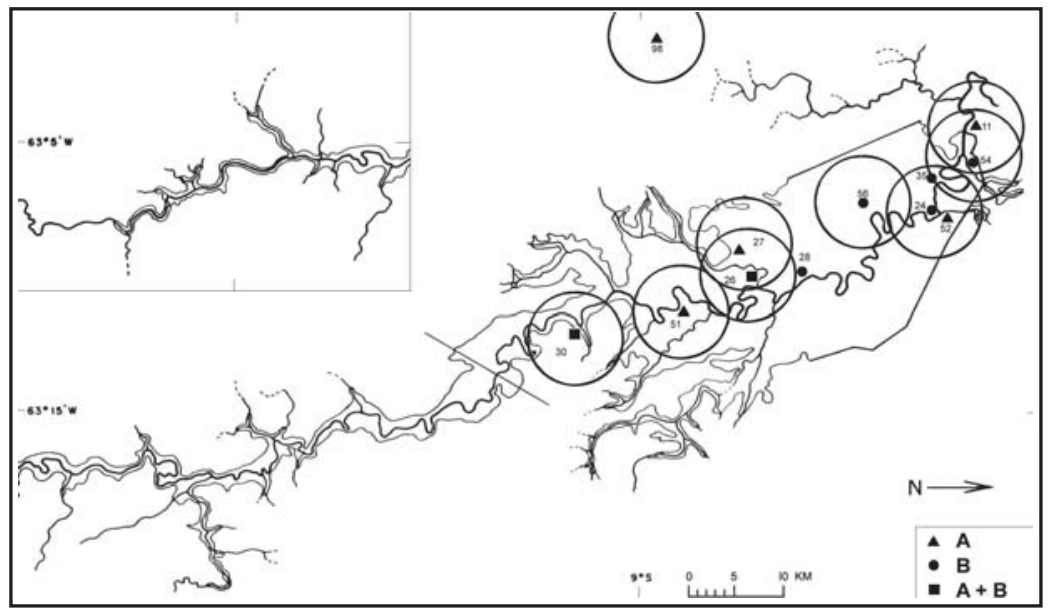

Figura 17.

Fase Jamarí: Hipotéticas zonas nucleares de caza.

Un estimado independiente de la capacidad de carga humana sostenible fue suministrado por los biólogos preocupados por el impacto creciente de la cacería comercial. La combinación de la edad de reproducción, número de progenie, longevidad, impacto de otros predadores, muerte natural y otras variables para cada especie permite calcular el número máximo de individuos por kilómetro cuadrado que se puede extraer sosteniblemente. Convirtiendo el resultado en biomasa y dividiendo el total por el requisito nutricional de un consumidor humano indica que una densidad humana mayor que $1 / \mathrm{km} 6$ no es sostenible y que la capacidad de carga verdadera es de $0.2 / \mathrm{km}^{2}$ o menos. No es probable que el acuerdo entre este resultado y las densidades de una docena de comunidades indígenas que mantienen adaptaciones sostenibles hoy sea una coincidencia (Fig 18) (Tabla1).

\section{Conclusión}

Mientras que nuestra reconstrucción se basa en patrones inferidos de la evidencia arqueológica e interpretados en el contexto de la teoría evolutiva, comportamiento etnográfico y limitaciones ambientales, la apremisa básica de los proponentes de poblaciones densas y sedentarias es aque los nativos amazónicos no se adaptaron a la naturaleza, sino que crearon el mundo que querían (Erickson 2003:457). En base a la hipótesis que las dimensiones de la terra preta son correlacionadas directamente con el tamaño y la duración de la aldea, ellos concluyen que las sociedades urbanas precolombinas inventaron métodos exitosos y eficientes para sostener poblaciones grandes y densas, producir excedentes y manejar basura (Erickson, 2003: 466-487). Aunque la terra preta se considera el producto de residencia permanente, la proponen como la solución para la agricultura intensiva. También 
Tabla 1

Densidad humana sostenible de grupos amazónicos

\begin{tabular}{l|l}
\hline $\begin{array}{c}\text { Densidad } \\
\text { (Estimada en base a la } \\
\text { cacería sostenible) }\end{array}$ & \multicolumn{1}{c}{ Referencia } \\
\hline$<1 / \mathrm{km}^{2}$ & Benett y Robinson, 2004: 14 y 24 \\
\hline $0.2 / \mathrm{km}^{2}$ & Hill y Padwe, 2000 \\
\hline$<1 / \mathrm{km}^{2}$ & Milner-Guiland et al, 2003: 351 \\
\hline $0.2 / \mathrm{km}^{2}$ & Phillips, 1993: 30-31 \\
\hline $0.087 / \mathrm{km}^{2}$ & Piaroa; Zent, 1998 \\
\hline $0.17 / \mathrm{km}^{2}$ & Achuar; Descola, 1981: 628 \\
\hline $0.9-1.8 / \mathrm{km}^{2}$ & Runa; Irvine: 225 \\
\hline$<1 \mathrm{milla}^{2}$ & Ye' Kwana, Yanomano; Hames, 1980: 33 \\
\hline $0.2 / \mathrm{km}^{2}$ & Siona-Secoya; Vickers, 1991: 77 \\
\hline $0.5 / \mathrm{km}^{2}$ & Huaorani; Mena et al, 2000: 58 \\
\hline $0.03 / \mathrm{km}^{2}$ & Aché; Hill y Padue, 2000: 56 \\
\hline $0.2 / \mathrm{km}^{2}$ & Tucanoan; Jackson, 1983: 21 \\
\hline $0.3 / \mathrm{km}^{2}$ & Machiguenga; Johnson, 1989: 215 \\
\hline $0.025 / \mathrm{km}^{2}$ & Waoarani; Yost y Kelly, 1983: 192 \\
\hline $1.5 / \mathrm{km}^{2}$ & Yukpa-Yuka; Ruddle, 1974: 28 \\
\hline $0.2 / \mathrm{km}^{2}$ & Ka' apor; Balée, 1994: 2 \\
\hline
\end{tabular}

asumen simultáneamente que el combustible fue abundante y accesible y que la selva primaria y secundaria alrededor de la aldea fue suplantada por cultivos y huertas. Estas y otras contradicciones tienen que ser resueltas antes que la existencia de poblaciones densas y sedentarias en la Amazonía pueda ser aceptada.

La reconstrucción del desarrollo cultural en la Amazonía representa un desafío excepcional para los arqueólogos, comparable al desafío enfrentado por los habitantes indígenas para conseguir una explotación sostenible de la complejidad ambiental. La escasez de la evidencia arqueológica hace necesaria la extracción de la mayor cantidad de información posible de las características de la cerámica y su distribución temporal y espacial. El análisis cuantitativo y la seriación constituyen un enfoque; los detalles de decoración y forma de vasija proveen un segundo; la teoría de la deriva evolutiva representa un tercero. Una comparación de las interpretaciones resultantes con la evidencia ambiental, biológica y climática llama la atención a correlaciones que no son evidentes desde la perspectiva local. Esperamos que los arqueólogos sean motivados en aceptar el desafío de refinar los métodos y elaborar las interpretaciones que hemos desarrollado. 


\section{Bibliografía}

ABBOT, David R.

2000 Ceramics and community organization among the Hohokam. Tucson, University of Arizona Press.

\section{ABRAMS, Elliot y RUE, David}

1988 «The causes and consequences of deforestation among the prehistoric Maya». In: Human Ecology 16 (4): 377-395.

\section{ABSY, Maria Lucía}

1982 «Quaternary palynological studies in the Amazon Basin». In: G.T. Prance (ed), Biological diversification in the tropics. New York: Columbia University Press, pp. 67-73.

\section{ALLEN, William L. y TIZON, Judy H.}

1973 «Land use patterns among the Campa of the Alto Pachitea, Peru». In: D.W. Lathrap y J. Douglas (eds.). Variation in anthropology. Urbana, Illinois Archaeological Survey, pp. 137-153.

\section{ALVARD, Michael S.; ROBINSON, John G.; REDFORD, Kent H. y KAPLAN, Hillard \\ 1997 «The sustainability of subsistence hunting in the Neotropics». In: Conservation Biology, 11: 977-982.}

\section{ALEXANDER, Michael ( ed.)}

1976 Discovering the New World, based on the works of Theodore de Bry. New York: Harper y Row.

ALVIM, P. de T.

1980 «Agricultural production potential of the Amazon region». In: F. BarbiraScassocchio (ed.). Land, people, and planning in contemporary Amazonía. Cambridge University Centre for Latin American Studies, Occasional Publication 3. Pp. 27-36.

\section{ARAUJO, Astolfo G. Mello y MARCELINO, José Carlos}

2003 «The role of armadillos in the movement of archaeological materials: an experimental approach». In: Geoarchaeology, 18: 433-460.

\section{ARHEM, Kaj}

1976 «Fishing and hunting among the Makuna». In: Gothenburg: Ethnographical Museum Annual Report, pp. 27-44. 
1981 «Makuna social organization: a study in descent, alliance and the formation of corporate groups in the north-western Amazon». In: Stockholm: Uppsala Studies in Cultural Anthropology, 4.

\section{ARNOLD, Dean E.}

1993 Ecology and ceramic production in an Andean community. New York: Cambridge University Press.

\section{ARNOLD III, Philip J.}

2003 «Early Formative pottery from the Tuxtla Mountains and implications for Gulf Olmec origins». In: Latin American Antiquity, 14: 29-46.

\section{BAKSH, Michael y JOHNSON, Allen}

1990 «Insurance policies among the Machiguenga: an ethnographic analysis of risk management in a non-western society». In: E. Cashdan (ed.), Risk and uncertainty in tribal and peasant economies, pp. 193-227. Boulder: Westview.

\section{BALÉE, William}

1994 Footprints in the forest: Ka=apor ethnobotanyBthe historical ecology of plant utilization by an Amazonian people. New York: Columbia University Press.

BARROS, Philip L.F. de

1982 «The effects of variable site occupation span on the results of frequency seriation». In: American Antiquity, 47: 91-315.

\section{BASSO, Ellen B.}

1973 The Kalapalo Indians of central Brazil. New York: Holt, Rinehart and Winston.

\section{BECKERMAN, Stephen}

1977 «The use of palms by the Barí Indians of the Maracaibo Basin». In: Principes, 21: $143-154$.

\section{BELLIER, Irene}

1991 El temblor y la luna: ensayo sobre las relaciones entre las mujeres y los hombres mai huna. Cayambe, Ediciones Abya-Yala.

\section{BENNETT, Elizabeth L. y ROBINSON, John G.}

2000 Hunting of wildlife in tropical forests: implications for biodiversity and forest peoples. Environmental Department Papers No. 76. Washington DC, The World Bank. 


\section{BETTEX, Albert}

1960 The discovery of the New World. New York, Simon and Schuster.

\section{BOWSER, Brenda J.}

2000 «From pottery to politics: an ethnoarchaeological study of political factionalism, ethnicity, and domestic pottery style in the Ecuadorian Amazon». In: Journal of Archaeological Method and Theory, 7: 219-248.

\section{BRUMBACH, Hetty Jo}

1985 "Ceramic analysis and the investigation of matrilocality at the Smith Mohawk village site». In: North American Archaeologist, 6: 341-355.

\section{BUTT, Audrey J.}

1977 «Land use and social organization of tropical forest peoples of the Guianas». In: J.P. Garlick y R.W. J. Keay, eds., Human ecology in the tropics, pp. 1-17. London, Taylor and Francis Ltd.

\section{CARVAJAL, Yesid; JIMÉNEZ, Henry y MATERÓN, Hernán}

1998 «Incidencia del fenómeno ENSO en la hidroclimatología del valle del río CaucaColombia». En: Bulletin d'Institut français d'Etudes andines, 27(3): 743-751.

\section{CARVALHO, José Cândido M.}

1952 «Notas de viagem ao Rio Negro. Publicações Avulsas». Rio de Janeiro, Museu Nacional.

\section{CERÓN SOLARTE, Benhur}

1988 «Los Awa-Cuaiquer: un grupo indígena de la selva pluvial del Pacífico Nariñense y el noroccidente ecuatoriano». Quito, Abya-Yala.

\section{CLARK, Kathleen E. y UHL, Christopher}

1984 «Deterioro de la vida de subsistencia tradicional en San Carlos de Rio Negro». En: Interciencia, 9: 358-365.

\section{COLSON, Audrey Butt}

1983-84 «The spatial component in the political structure of the Carib speakers of the Guiana highlands: Kapon and Pemon». En: Antropológica, 59-62: 73-124.

\section{CONKLIN, Beth A.}

2001 Consuming grief: compassionate cannibalism in an Amazonían society. Austin, University of Texas Press. 


\section{CORREA, François}

1987 «Indígenas horticultores del Vaupés». En: Introducción a la Colombia Amerindia, pp. 109-122. Bogotá, Instituto Colombiano de Antropología.

\section{DESCOLA, Philippe}

1981 «From scattered to nucleated settlement: a process of socioeconomic change among the Achuar». In: N. Whitten (ed.), Cultural transformations and ethnicity in modern Ecuador, pp. 614-646. Urbana, University of Illinois Press.

1994 «Homeostasis as a cultural system: the Jivaro case». In: A.C. Roosevelt (ed.), Amazonían Indians, pp. 203-224. Tucson, University of Arizona Press.

1996 The spears of twilight: life and death in the Amazon jungle. New York, The New Press.

\section{DUFF, Andrew I.}

1996 «Ceramic micro-seriation: types or attributes?». In: American Antiquity, 61: 89-101.

\section{EDEN, Michael J.}

1974 «Ecological aspects of development among Piaroa and Guahibo Indians of the upper Orinoco basin». In: Antropológica, 39: 25-56.

\section{ERICKSON, Clark}

2003 «Historical ecology and future explorations». In: J. Lehmann et al (eds.), Amazonian dark earths, pp. 455-500. Dordrecht, Kluwer Academic Publishers.

\section{EVANS, Clifford y MEGGERS, Betty J.}

1960 Archeological investigations in British Guiana. Bureau of American Ethnology Bul. 177. Washington DC, Smithsonian Institution.

FEARNSIDE, $P$.

1987 «Rethinking continuous cultivation in Amazonía». In: BioScience, 37: 209-214.

\section{FOSTER, Robin B.}

1982 «Famine on Barro Colorado island». In: E.G. Leigh Jr. et al (eds.), The ecology of a tropical forest, pp. 201-212. Washington DC, Smithsonian Institution.

\section{GALLOIS, Dominique}

1981 «Os Waiapi e seu território». En: Bol. Mus. Par. Em. Goeldi, Antropologia, № 80.

\section{GOLDMAN, Irving}

1979 «The Cubeo, Indians of the northwest Amazon». Second Edition. Urbana, University of Illinois Press. 


\section{GOOD, Kenneth R.}

1987 «Limiting factors in Amazonían ecology». In: Marvin Harris y Eric B. Ross (eds.), Food and evolution, pp. 407-421. Philadelphia, Temple University Press.

\section{GRAGSON, Tom L.}

1995 «Pumé exploitation of Mauritia Flexuosa (Palmae) in the Llanos de Venezuela». In: Journal of Ethnobiology, 15: 177-188.

\section{GRAVES, Michael W.}

1985 «Ceramic design variation within a Kalinga village: temporal and spatial processes». In: B.A. Nelson (ed.), Decoding prehistoric ceramics, pp. 9-34. Carbondale, Southern Illinois University Press.

\section{GROSS, Daniel R.}

1975 «Protein capture and cultural development in the Amazon basin». In: American Anthropologist, 77: 526-549.

1983 «Village movement in relation to resources in Amazonía». In: R. B. Hames y W.T. Vickers (eds.), Adaptive responses of native Amazoníans, pp. 429-449. New York, Academic Press.

\section{HALSTEAD, Paul y O’SHEA, John}

1989 «Introduction: cultural responses to risk and uncertainty». In: P. Halstead y J. O’Shea (eds.), Bad year economics, pp. 1-7. Cambridge, Cambridge University Press.

\section{HAMES, Raymond B.}

1980 «Game depletion and hunting zone rotation among the Ye'kwana and Yanomamo of Amazonas, Venezuela». In: Working Papers on South American Indians, 2: 31-66. Bennington VT, Bennington College.

\section{HECKENBERGER, Michael J.}

1992 «A conquista da Amazônia». Em: Ciência Hoje, 15: 62-67.

\section{HECKENBERGER, Michael J., KUIKURO, Afukaka, et al}

2003 «Amazonía 1492: pristine forest or cultural parkland?». In: Science 301: $1710-1714$.

\section{HENLEY, Paul}

1982 The Panare: tradition and change on the Amazon frontier. New Haven, Yale University Press. 


\section{HILL, Jonathan}

1984 «Social equality and ritual hierarchy: the Arawakan Wakuénai of Venezuela». In: American Ethnologist, 11: 528-544.

\section{HILL, Kim y PADWE, Jonathan}

2000 «Sustainability of Aché hunting in the Mbaracayu Reserve, Paraguay».In: J.G. Robinson y E.L. Bennett (eds.), Hunting for sustainability in tropical forests, pp. 79-105. New York, Columbia University Press.

\section{HODGES, H.W.M.}

1965 «Aspects of pottery in temperate Europe before the Roman Empire». In: F.R. Matson (ed.), Ceramics and man, pp. 114-123. Viking Fund Publications in Anthropology 41. Chicago, Aldine.

\section{IRVINE, Dominique}

1989 «Succession management and resource distribution in an Amazonían rain forest. Advances». In: Economic Botany, 7: 223-237. New York Botanical Garden.

\section{JACKSON, Jean E.}

1983 The fish people. Cambridge, Cambridge University Press.

\section{JOHNSON, Allen}

1989 «How the Machiguenga manage resources: conservation of exploitation of nature?». In: Advances in Economic Botany, 7: 213-222. New York Botanical Garden.

\section{KANE, Joe}

1995 Savages. New York, Knopf.

\section{KELLY, Robert L.}

1995 The foraging spectrum. Washington DC, Smithsonian Institution Press.

\section{KERN, Dirse Clara, et al}

2003 «Distribution of Amazonían dark earths in the Brazilian Amazon». In: Amazonían dark earths, J. Lehmann (ed.), pp. 51-75. Dordrecht, Kluwer Academic Publishers.

\section{LEEUWENBERG, Frans J. y ROBINSON, John G.}

2000 «Traditional management of hunting by a Xavante community in central Brazil: the search for sustainability». In: J.B. Robinson y E.L. Bennett (eds.), Hunting for sustainability in tropical forests, pp. 375-394. New York, Columbia University Press. 


\section{LEOPOLDO, Paulo Rodolfo}

2000 «Ciclo hidrológico em bacias experimentais da Amazônia central». Em: E. Salati, M.L.Absy y R.L. Victória (Eds), Amazônia: um ecosistema em transformação, pp. 87-118. Manaus, INPA.

\section{LIPO, Carl P., MADSEN, Mark E., DUNNELL, Robert y HUNT, Tim}

1997 «Population structure, cultural transmission, and frequency seriation». In: Journal of Anthropological Archaeology, 16: 301-333.

\section{LIZOT, Jacques}

1974 «El río de los Periquitos: breve relato de un viaje entre los Yanomami del Alto Siapa». En: Antropológica, 37: 323.

1980 «La agricultura Yanomama». En: Antropológica, 53: 3-93.

1984 Les Yanomami centraux. Paris, Ecole des Hautes Etudes en Sciences Sociales.

1993 «Yanomami natural resources use: an inclusive cultural strategy». In: A. Hladik et al (eds.), Tropical forests, people and foods, pp. 479-486. Paris, UNESCO.

\section{LONGACRE, William A.}

1964 «Sociological implications of the ceramic analysis». In: Fieldiana Anthropology, 55: 155-170.

1985 «Pottery use-life among the Kalinga, Northern Luzon, the Philippines. IN: B.A. Nelson (ed.), Decoding prehistoric ceramics, pp. 334-346. Carbondale, Southern Illinois University Press.

\section{LOPINOT, Neal H. y WOODS, William I.}

1993 «Wood overexploitation and the collapse of Cahokia». In: C. Margaret Scarry, ed., Foraging and farming in the eastern woodlands, pp. 206-231. Gainesville, University Press of Florida.

\section{MEGGERS, Betty J.}

1990 «Reconstrução do comportamento locacional pré-histórico na Amazônia». In: Boletim do Museu Paraense Emílio Goeldi, Antropologia, 6: 183-203.

1994 «Archeological evidence for the impact of mega-Niño events on Amazonía during the past two millennia». In: Climatic Change, 28: 321-338.

1996 Amazonía: man and culture in a counterfeit paradise. Washington DC, Smithsonian Institution Press.

2001 «The continuing quest for El Dorado: round two». In: Latin American Antiquity, 12: 304-325.

1999 La utilidad de secuencias cerámicas seriadas para inferior conducta social prehistórica. El Caribe Arqueológico 3:2-19.

2007 «Sustainable intensive exploitation of Amazonia: cultural, environmental, and geopolitical perspectives». The World System and the Earth System, Alf 
Hornborg \& Carole L. Crumley, eds., pp 195-209. Walnut Creek CA, Left Coast Press Inc. Meggers, Betty J. y Eurico Th. Miller

2006 «Evidencia arqueológica para el comportamiento social y habitacional en la Amazonía prehistórica». Pueblos y Paisajes Antiguos de la Selva Amazónica, Gaspar Morcote Ríos, Santiago Mora Camargo y Carlos Franky Calvo, eds. Pp. 325-348. Bogotá, Universidad Nacional de Colombia.

MENA, Patricio, STALLINGS, Jody R., REGALADO, Jhanira y CUEVA L., Ruben 2000 «The sustainability of current hunting practices by the Huaorani». In: J.G. Robinson y E.L. Bennett (eds.), Hunting for sustainability in tropical forests, pp. 57-78. New York, Columbia University Press.

\section{MERONA, B. de}

1990 «Amazon fisheries: general characteristics based on two case studies». In: Interciencia, 15: 461-475.

\section{MIGLIAZZA, Ernest C.}

1982 «Linguistic prehistory and the refuge model in Amazonía». In: G.T. Prance (ed.), Biological diversification in the tropics, pp. 497-519. New York, Columbia University Press.

\section{MILLER, Eurico Th.}

1983 História da cultura indígena do alto Médio-Guaporé (Rondônia e Mato Grosso). Tese de Mestrado, Pontificia Universidade Católica do Rio Grande do Sul, Porto Alegre.

1992 «Adaptação agrícola pré-histórica no Alto Rio Madeira». In: B.J. Meggers (ed.), Prehistoria sudamericana, pp. 219-229. Washington, Taraxacum.

1999 «A limitação ambiental como barreira à transposição do período formativo no Brasil: tecnologia, produção de alimentos e formação de aldeias no sudoeste da Amazônia». In: P. Ledergerber-Crespo (ed.), Formativo sudamericano, pp. 331-339. Quito, Abya Yala.

\section{MILLER, Eurico Th. et al}

1992 Arqueologia nos empreendimentos hidrelétricos da Eletronorte: resultados preliminares. Brasília, Centrais Elétricas do Norte do Brasil S.A.

\section{MILNER, George R.}

1998 The Cahokia chiefdom: the archaeology of a Mississippian society. Washington, Smithsonian Institution Press. 


\section{MILNER-GULLAND, L.J. y AKCAKAYA, H. Resit}

2001 «Sustainability indices for exploited populations». En: Trends in Ecology and Evolution, 16: 686-692.

\section{MILNER-GULLAND, E.J., BENNETT, Elizabeth L.}

2003 «Wild meat: the bigger picture». En: Trends in Ecology and Evolution, 18: 351-357.

\section{MORÁN, Emilio F.}

1984 «Amazon Basin colonization». En: Interciencia, 9: 377-385.

\section{MURPHY, Robert F. y QUAIN, Buell}

1955 The Trumai Indians of Central Brazil. Monographs of the American Ethnological Society 24. Seattle.

\section{MYERS, Thomas P., DENEVAN, William M., et al}

2003 «Historical perspectives on Amazonían dark earths». En: J. Lehmann et al (eds.), Amazonían dark earths, pp. 15-24. Dordrecht, Kluwer Academic Publishers.

\section{NEFF, Hector}

1992 «Ceramics and evolution». En: Archaeological Method and Theory, 4: 141-193.

NEVES, Eduardo G., PETERSON, James B., et al

2003 «Historical and socio-cultural origins of Amazonían dark earths». En: J. Lehmann et al (eds.), Amazonían dark earths, pp. 29-50. Dordrecht, Kluwer Academic Publishers.

\section{NAIKIAI, Verónica}

1987 «El uso del ecosistema en el antiguo pueblo Shuar». En: José Juncosa (ed.), Los guardianes de la tierra, pp. 9-33. Quito, AbyaYala.

\section{NORTHCLIFF, Stephen y DIAS, Antonio D.C.P.}

1988 «The change in soil physical conditions resulting from forest clearance in the humid tropics». En: Journal of Biogeography, 15: 61-66.

\section{OLSEN, Kenneth M. y SCHAAL, Barbara A.}

1999 «Evidence on the origin of cassava: phylogeography of Manihot esculenta». En: Proceedings National Academy of Science, 96: 5586-5591

2001 «Micosatellite variation in cassava (Manihot esculenta, Euphorbiaceae) and its wild relatives: further evidence for a southern Amazonían origin of domestication». En: American Journal of Botany, 88: 131-142. 
PEREIRA, Francisco y SALINAS, José G.

1982 «General evaluation of the agricultural potential of the Bolivian Amazon». En: S.B. Hecht (ed.), Amazonía: agriculture and land-use research, pp. 17-31. Cali, CIAT.

PERES, Charles A.

2000 «Evaluating the impact and sustainability of subsistence hunting at multiple Amazonían forest sites». En: J.G. Robinson y E.L. Bennett (eds.), Hunting for sustainability in tropical forests, pp. 31-56. New York, Columbia University Press.

\section{PEROTA, Celso}

1992 «Adaptação agrícola no baixo Xingu». En: B.J. Meggers (ed.), Prehistoria sudamericana, pp. 211-218. Washington, Taraxacum.

\section{PEROTA, Celso y BOTELHO, W.C.}

1987 «Sambaquis fluviais no baixo Xingu». En: Anais do 11 Congresso da Associação Brasileira de Estudios do Quaternário, pp. 487-491. Porto Alegre.

\section{PHILLIPS, Oliver}

1993 «The potential for harvesting fruits in tropical rainforests: new data from Amazonían Peru». En: Biodiversity and Conservation, 2: 18-38.

\section{PRICE, David}

1990 «Our readers write». En: Current Anthropology, 31: 386.

\section{POSEY, Darrell A.}

1983 «Indigenous ecological knowledge and development of the Amazon». En: Emilio F. Moran (ed.), The dilemma of Amazonian development, pp. 225-257. Boulder, Westview.

\section{PURDY, Barbara A.}

1996 How to do archaeology the right way. Gainesville, University Press of Florida.

\section{REICHEL-DOLMATOFF, Gerardo}

1976 «Cosmology as ecological analysis: a view from the rain forest». En: Man, 11: 307-318.

1990 «Algunos conceptos de los indios Desana del Vaupés sobre manejo ecológico». En: F. Correa (ed.), La selva humanizada, pp. 35-41.Bogotá, ICANFEN-Cerec, Serie Amerindia No.1.

1996 The forest within: the world-view of the Tukano Amazonian Indians. Devon, Themis Books. 


\section{REINA, Ruben E. y HILL, Robert}

1978 The traditional pottery of Guatemala. Austin, University of Texas Press.

\section{ROBINSON, John G.}

2000 «Calculating maximum sustainable harvests and percentage offtakes». En: J.G. Robinson y E.L. Bennett (eds.), Hunting for sustainability in tropical forests, pp. 521-524. New York, Columbia University Press.

\section{ROBINSON, John G. y BENNETT, Elizabeth L.}

2000 «Carrying capacity limits to sustainable hunting in tropical forests». En: J.G. Robinson y E.L. Bennett (eds.), Hunting for sustainability in tropical forests, pp. 13-30. New York, Columbia University Press.

\section{ROE, Peter G.}

1981 «Aboriginal tourists and artistic exchange between the Pisquibo and the Shipibo: A Trade ware in an ethnographic setting». En: P.D.Francis, F.J.Kense, y P.G.Duke (eds.), Networks of the past, pp. 61-84. Calgary, Univ. of Calgary Archaeological Assn.

\section{ROOSEVELT, Anna C.}

1993 «The rise and fall of Amazonían chiefdoms». En: L'Homme, 33(126-128): 255-283.

\section{RUDDLE, Kenneth}

1974 «The Yukpa cultivation system: a study of shifting cultivation in Colombia and Venezuela». En: Ibero-Americana, 52. Berkeley, University of California Press.

\section{SALDARRIAGA, J.G. y D.C. West}

1986 «Holocene fires in the northern Amazon Basin». En: Quaternary Research, 26: 358-366.

\section{SCHULTES, Richard Evans}

1977 «Diversas plantas comestíveis nativas do noreste da Amazônia». En: Acta Amazônica, 7: 317-327.

\section{SEEGER, Anthony}

1981 Nature and society in central Brazil: the Suya Indians of Mato Grosso. Cambridge, Harvard University Press.

\section{SIMOES, Mário F.}

1974 «Contribuição à arqueologia dos arredores do baixo Rio Negro, Amazonas». En: Publicações Avulsas, 26: 165-200. Belém, Museu Paraense Emílio Goeldi. 


\section{SIMOES, Mário F. y ARAUJO-COSTA, Fernanda}

1987 «Pesquisas arqueológicas no baixo rio Tocantins (Pará)». En: Revista de Arqueologia, 4: 11-27.

\section{SIMOES, Mário F. y GENTIL CORREA, Conceição}

1987 «Pesquisas arqueológicas no baixo Uatumã-Jatapu (Amazonas)». En: Revista de Arqueologia, 4: 29-48.

\section{SIMOES, Mário F. y MACHADO, Ana Lucia}

1987 «Pesquisas arqueológicas no lago de Silves (Amazonas)». En: Revista de Arqueologia, 4: 49-82.

SIMOES, Mário F. y KALKMANN, Ana Lúcia M.

1987 «Pesquisas arqueológicas no Médio Rio Negro (Amazonas)». En: Revista de Arqueologia, 4: 83-116.

SIMOES, Mário F. y LOPES, Daniel F.

1987 «Pesquisas arqueológicas no baixo/médio Rio Madeira (Amazonas)». En: Revista de Arqueologia, 4: 117-134.

SLADE, N. A. et al

1998 «Alternatives to Robinson and Redford's method of assessing overharvest from incomplete demographic data». En: Conservation Biology, 12: 148-155.

SPONSEL, Leslie E.

1986 La cacería de los Yekuana bajo una perspectiva ecológica. Caracas, Universidad Católica Andres Bello.

STAHL, Peter W.

2002 «Paradigms in paradise: revising standard Amazonían prehistory». En: The Review of Archaeology, 23: 39-51.

STAHL, Peter W. y ZEIDLER, James

1990 «Differential bone-refuse accumulation in food-preparation and traffic areas on an early Ecuadorian house floor». En: Latin American Antiquity, 1: 150-169.

\section{STEARMAN, Allyn Maclean}

1990 "The effects of settler incursion on fish and game resources of the Yuquí, a native American Society of eastern Bolivia». En: Human Organization, 49: 373-385. 


\section{TRIANA, Gloria}

1987 «Puinave». En: Introducción a la Colombia Amerindia, pp. 97-107. Bogotá, Instituto de Antropología.

\section{URIARTE, Luis}

1985 «Los nativos y su territorio: el caso de los Jívaro Achuara en la Amazonía peruana». En: Amazonía Peruana, 11: 39-64.

\section{VICKERS, William T.}

1983 «The territorial dimensions of Siona-Secoya and Encabello adaptation». En: R.B. Hames y W.T. Vickers, Adaptive responses of native Amazoníans, pp. 451478. New York, Academic Press.

1988 «Game depletion hypothesis of Amazonían adaptation: data from a native community». En: Science, 239: 1521-1522.

1989 «Patterns of foraging and gardening in a semi-sedentary Amazonían community. En: S. Kent (ed.), Farmers as hunters, pp. 46-59. Cambridge University Press.

1991 «Hunting yields and game composition over ten years in an Amazon Indian territory». En: J.G. Robinson y K.H. Redford (eds.), Neotropical wildlife use and conservation, pp. 53-81. Chicago, University of Chicago Press.

WAGLEY, Charles

1977 Welcome of tears: the Tapirapé Indians of central Brazil. New York, Oxford University Press.

\section{WEBSTER, David}

1997 «City-states of the Maya». En: D.L. Nichols y T.H. Charlton (eds.), The archaeology of city-states, pp. 135-154. Washington, Smithsonian Institution Press.

\section{WEISCHET, Wolfgang and CAVIEDES, Cesar N.}

1993 The persisting ecological constraints of tropical agriculture. New York, John Wiley and Sons.

\section{WHITEHEAD, Neil L.}

1994 «The ancient amerindian polities of the Amazon, the Orinoco, and the Atlantic coast: a preliminary analysis of their passage from antiquity to extinction». En: A.C.Roosevelt (ed.), Amazonían Indians, pp. 33-53. Tucson, University of Arizona Press.

WRIGHT, S. Joseph, CARRASCO, Claudio, CALDERÓN, Osvaldo y PATON, Steven 1999 «The El Niño Southern Oscillation, variable fruit production, and famine in a tropical forest». En: Ecology, 80: 1632-1647. 


\section{WÜST, Irmhild}

1987-89 «A pesquisa arqueológica e etnoarqueológica na parte central do territorio Bororo, Mato Grosso - primeiros resultados». En: Revista de Antropologia, 30-32:21-35.

1994 «The eastern Bororo from an archaeological perspective». En: A.C. Roosevelt (ed.), Amazonían Indians, pp. 315-342. Tucson, University of Arizona Press.

\section{YOST, James A.}

1981 «Twenty years of contact: the mechanisms of change in Wao (Auca) culture». En: N.E. Whitten Jr. (ed.), Cultural transformations and ethnicity in modern Ecuador, pp. 677-704. Urbana, University of Illinois Press.

\section{Yost, James A. y Kelley, Patricia M.}

1983 «Shotguns, blowguns, and spears: the analysis of technological efficiency». En: R.B.Hames y W.T. Vickers (eds.), Adaptive responses of native Amazoníans, pp. 189-224. New York, Academic Press.

\section{ZEIDLER, James A.}

1983 «La etnoarqueología de una vivienda Achuar y sus implicaciones arqueológicas». En: Miscelánea Antropológica Ecuatoriana, 3: 155-193.

\section{ZENT, Egleé y ZENT, Stanford}

2002 «Impactos ambientales generadores de biodiversidad: conductas ecológicas de los Hoti de la Sierra Maigualida, Amazonas, Venezuela». En: Interciencia, 27: 9-20.

\section{ZENT, Stanford}

1992 Historical and ethnographic ecology of the upper Cuao River Wõthihã: clues for an interpretation of native Guianese social organization. PhD Dissertation, Columbia University, New York.

1998 «Independent yet interdependent Aisode@: the historical ecology of traditional Piaroa settlement pattern». En: W. Balée (ed.), Advances in historical ecology, pp. 251-285. New York, Columbia University Press. 